University of Rhode Island

DigitalCommons@URI

Open Access Master's Theses

2002

\title{
Evaluation of Several Metrics of Benthic Macroinvertibrates
}

Scott Lynch

University of Rhode Island

Follow this and additional works at: https://digitalcommons.uri.edu/theses

\section{Recommended Citation}

Lynch, Scott, "Evaluation of Several Metrics of Benthic Macroinvertibrates" (2002). Open Access Master's Theses. Paper 1150.

https://digitalcommons.uri.edu/theses/1150

This Thesis is brought to you for free and open access by DigitalCommons@URI. It has been accepted for inclusion in Open Access Master's Theses by an authorized administrator of DigitalCommons@URI. For more information, please contact digitalcommons@etal.uri.edu. 


\section{EVALUATION OF SEVERAL METRICS OF BENTHIC}

\section{MACROINVERTEBRATES}

BY

SCOTT LYNCH

A THESIS SUBMITTED IN PARTIAL FULFILLMENT OF THE

REQIREMENTS FOR THE DEGREE OF

MASTER OF SCIENCE

IN

STATISTICS

UNIVERSITY OF RHODE ISLAND

2002 


\section{MASTER OF SCIENCE THESIS}

OF

SCOTT LYNCH

APPROVED:

Thesis Committee:

Major Professor
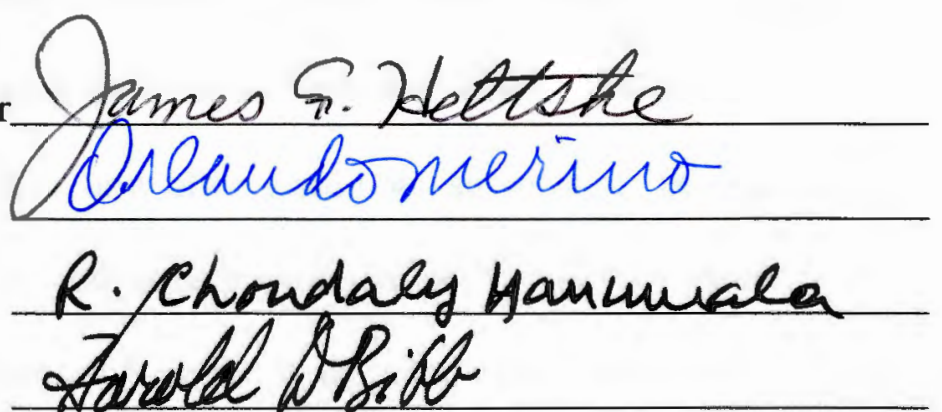

DEAN OF THE GRADUATE SCHOOL

UNIVERSITY OF RHODE ISLAND 


\section{ABSTRACT}

This study simulates individuals that are modeled after benthic macroinvertebrate populations from streams in Vermont. Using the EPA biological scoring criteria for assessing the overall condition of streams, we analyzed two metrics (parameters) associated with these criteria. The two metrics, taxa richness and Community Loss Index (CLI) have "critical" values that were set by the EPA. These "critical" values although well established are not be justified statistically. In order to analyze these "critical" values, we analyzed three different options: sample size, taxonomic level, and changing the "critical" value to achieve our goal.

We asked the question, how often would two samples of 100 organisms, which are both taken from the same reference population, be considered different? By simulating two different samples from the same population 100 times, we could obtain an estimate of percentage of times one would conclude the population to be different. Generally, the best results occurred at the genus level with a slight modification to the "critical" value and a sample size of 100 for the taxa richness metric. For CLI, the genus level with a sample size of 200 and no "critical" value change produced the best results. 


\section{ACKNOWLEDGEMENTS}

In completing this thesis, I would like to thank Dr. James Heltshe for the opportunity to work with him including his efforts, time, patience and guidance. I would also like to thank my committee members, Dr. Choudary Hanumara, Dr.

Orlando Merino and Dr. David Shao for their time and suggestions. I would also like to thank my parents for their encouragement and support. Lastly, I would like to thank my wife for her love and always being there for me. 


\section{PREFACE}

This thesis was prepared in standard format. It contains an analysis of two different metrics used in stream sampling for benthic macroinvertebrates. Computer simulations were applied to mimic different populations of streams in order to determine the effectiveness of each metric. 


\section{TABLE OF CONTENTS}

\section{Page}

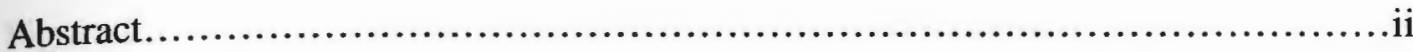

Acknowledgments...............................................................

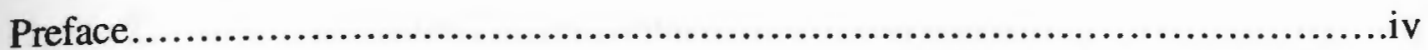

Table of Contents............................................................

List of Figures.................................................................

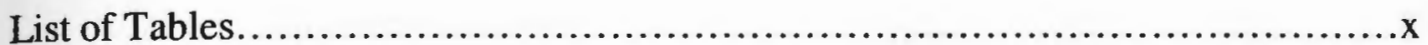

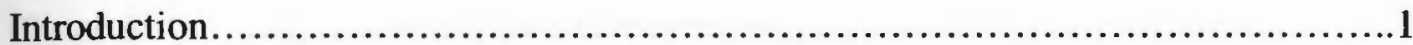

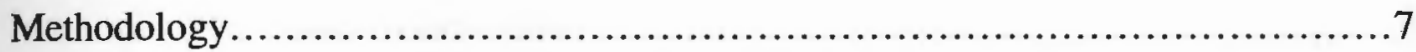

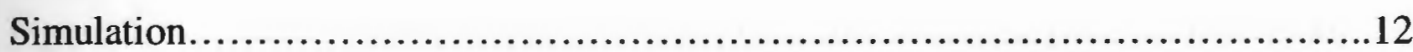

Results.............................................................................

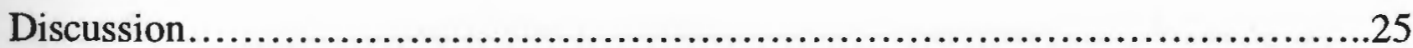

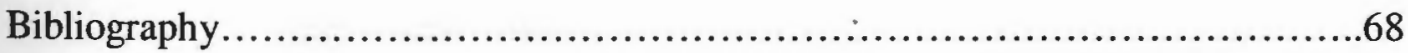




\section{LIST OF FIGURES}

Page

Figure 1. Genus Level: True Richness vs. Alpha (Richness)

Sample size $=100$

Figure 2. Genus Level: True Richness vs. Alpha (CLI)

Sample size $=100$

Figure 3. Genus Level: True Evenness vs. Alpha (Richness)

Sample size $=100$

Figure 4. Genus Level: True Evenness vs. Alpha (CLI)

Sample size $=100$ .34

Figure 5. Genus Level: True Richness vs. True Evenness (Richness Alpha)

Sample size $=100$

Figure 6. Genus Level: True Richness vs. True Evenness (CLI Alpha)

Sample size $=100$ .36

Figure 7. Genus Level: True Richness vs. Alpha (Richness)

Sample size $=200$

Figure 8. Genus Level: True Richness vs. Alpha (CLI)

Sample size $=200$ .38

Figure 9. Genus Level: True Evenness vs. Alpha (Richness)

Sample size $=200$ 
Figure 10. Genus Level: True Evenness vs. Alpha (CLI)

$$
\text { Sample size }=200 \text {. }
$$

Figure 11. Genus Level: True Richness vs. True Evenness (Richness Alpha)

Sample size $=200$.

Figure 12. Genus Level: True Richness vs. True Evenness (CLI Alpha)

Sample size $=200$.

Figure 13. Family Level: True Richness vs. Alpha (Richness)

Sample size $=100$.

Figure 14. Family Level: True Richness vs. Alpha (CLI)

Sample size $=100$.

Figure 15. Family Level: True Evenness vs. Alpha (Richness)

Sample size $=100$

Figure 16. Family Level: True Evenness vs. Alpha (CLI)

Sample size $=100$.

Figure 17. Family Level: True Richness vs. True Evenness (Richness Alpha)

Sample size $=100$.

Figure 18. Family Level: True Richness vs. True Evenness (CLI Alpha)

Sample size $=100$. .48

Figure 19. Family Level: True Richness vs. Alpha (Richness)

Sample size $=200$. 49 
Figure 20. Family Level: True Evenness vs. Alpha (Richness)

Sample size $=200$ 50

Figure 21. Family Level: True Richness vs. True Evenness (Richness Alpha)

Sample size $=200$

Figure 22. Family Level: True Richness vs. Alpha (Richness)

Sample size $=300$

Figure 23. Family Level: True Evenness vs. Alpha (Richness)

Sample size $=300$

Figure 24. Genus Level: True Richness vs. Alpha (Richness) $=0.75$

Sample size $=100$

Figure 25. Genus Level: True Evenness vs. Alpha (Richness) $=0.75$

Sample size $=100$ .55

Figure 26. Genus Level: True Richness vs. Alpha (CLI) $=0.55$

Sample size $=100$ .56

Figure 27. Genus Level: True Evenness vs. Alpha (CLI) $=0.55$

Sample size $=100$ .57

Figure 28. Genus Level: True Richness vs. Alpha (CLI) $=0.60$

Sample size $=100$ .58

Figure 29. Genus Level: True Evenness vs. Alpha (CLI) $=0.60$

Sample size $=100$. .59

Figure 30. Family Level: True Richness vs. Alpha (Richness) $=0.75$

Sample size $=100$ 
Figure 31. Family Level: True Evenness vs. Alpha (Richness) $=0.75$

Sample size $=100$

Figure 32. Family Level: True Richness vs. Alpha (Richness) $=0.70$

Sample size $=100$.

Figure 33. Family Level: True Evenness vs. Alpha (Richness) $=0.70$

Sample size $=100$.

Figure 34. Family Level: True Richness vs. Alpha (CLI) $=0.55$

Sample size $=100$

Figure 35. Family Level: True Evenness vs. Alpha (CLI) $=0.55$

Sample size $=100$

Figure 36. Family Level: True Richness vs. Alpha $(\mathrm{CLI})=0.60$

Sample size $=100$ .66

Figure 37. Family Level: True Evenness vs. Alpha (CLI) $=0.60$

Sample size $=100$ 


\section{LIST OF TABLES}

Page

Table 1. Biological Condition Scoring Criteria...............................5

Table 2. Summary Totals for Richness ....................................26

Table 3. Summary Totals for CLI........................................27 


\section{Introduction}

Assessing the overall quality of streams and rivers is an important aspect of determining how the environment is changing over the course of time. There are many different methods, which are utilized for examining the environmental factors in water quality. However, one of the most important, if not the best indicators, is the fertility and dispersion of benthic macroinvertebrates. The term 'benthic' means 'bottom-living' and indicates that these organisms usually inhabit bottom substrates for at least part of their life cycle; the prefix 'macro' indicates that these organisms are retained by

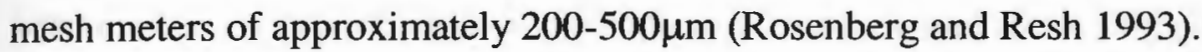

Benthic macroinvertebrate species are differentially sensitive to many biotic and abiotic factors in their environment. Consequently, macroinvertebrate community structure has commonly been used as an indicator of the condition of an aquatic system (Armitage $e t$ al, 1983). These organisms present several advantages to biologists when discovering conditions of streams. The primary benefit is the fact that they are good indicators of localized conditions, because many benthic macroinvertebrates have limited migration patterns or a sessile mode of life, they are particularly well suited for assessing site-specific impacts (EPA 1999). The main rationale for consideration is the expectation that these organisms integrate variations in water quality and therefore have the ability to reflect transient, adverse conditions that can be missed by sporadic sampling of chemistry (Isaac and Szal 2000). In addition, macroinvertebrates integrate the effects of short-term environmental variations, mostly because of 
their complex life cycle of approximately one year or more. Sensitive life stages will respond quickly to stress, but the overall community will tend to respond more slowly (EPA 1989). Another consideration is the fact that sampling is relatively easy, requiring only a few people and more importantly, has a minimal detrimental effect on the resident biota. Also, identification of benthic macroinvertebrates can be achieved with only a minimal effort. In fact, an experienced biologist can readily classify macroinvertebrates to the family level and many "intolerant" organisms to lower levels as well. Lastly, benthic macroinvertebrate assemblages are made up of species that constitute a broad range of trophic levels and pollution tolerances, thus providing strong information for interpreting cumulative effects (EPA 1989). With the growing problem of pollution levels evident globally, there has been a recent surge in the importance of benthic macroinvertebrates. Detailed observations as well as careful monitoring of these organisms in their habitat have become extremely important. There has also been an increasing attempt to develop proper methods of sampling macroinvertebrates. The Environmental Protection Agency (EPA) has become the greatest influence towards regulating and enforcing methods to maintaining safe conditions of water quality. In fact, since 1989 the EPA has produced substantial guidance and documentation on both bioassessment strategies and implementation policy on biological surveys and criteria for water resource programs (EPA 1999). Since the invoking of the Clean Water Act, it has become necessary to implement a methodical means of monitoring of streams that was greatly lacking across the country. It 
was further recognized that it was crucial to collect, compile, analyze and interpret environmental data rapidly to facilitate management decisions and resultant actions for control and/or mitigation of impairment (EPA 1989). Thus, the concept of Rapid Bioassessment Protocols (RBP's) was established. Originally, these RBP's had five main goals to achieve. First, the procedures for biological surveys needed to be cost-effective as well as scientifically valid. Second, provisions for multiple site investigations in a field season needed to be made. Next, quick turn-around of results for management decisions was also necessary. Also, scientific reports, which are easily translated to management and the public, had to be written. Finally, the procedures that were to be established needed to be harmless to the environment. After these concepts were established, it was determined that these protocols were to be centered on benthic macroinvertebrates. Since the original RBP's were created, there have been several revisions made to sharpen the precision of testing and sampling of these organisms due to advances in technology. Actually, there were three different protocols developed for benthic macroinvertebrates, each one with a more intensive evaluation than the previous. These protocols have three components in common, water quality/physical characteristics, habitat assessment, and a biosurvey. Upon gathering observations made in the assessment of habitat, water quality, physical characteristics, and the qualitative biosurvey, an investigator can conclude whether a site is impaired. The data analysis scheme used integrates several community, population, and functional parameters into a single 
evaluation of biotic integrity (EPA 1989). Each component, or metric, depicts a different component of community structure and has a specific range of sensitivity to pollution stress. This integrated approach provides more assurance of a valid assessment because a variety of parameters are evaluated (EPA 1989). There are at least 8 different metrics that are considered to be important in the analysis of the status of a biological macroinvertebrate community. Of these metrics, there are two which will be evaluated, taxa (species) richness and community loss index (CLI).

The oldest and the simplest concept of species diversity is species richness: the number of species in the community (Krebs, 1999). This measure reflects the health of a community through a measurement of the variety of taxa present (EPA 1989). Basically, taxa richness increases as the quality of water increases. Developed by Courtemanche and Davies (1987), the Community Loss Index (CLI) shows a dissimilarity with values increasing as the degree of dissimilarity from a reference station increases (EPA 1989). It can also be used to describe a difference between a reference station and a station of comparison. Even though there are other metrics, these two metrics help to give a well rounded look at the community, given the data that was presented. 
Table 1.

\section{Biological Condition Scoring Criteria}

\begin{tabular}{lllll}
\hline Metric & $\mathbf{6}$ & $\mathbf{4}$ & $\mathbf{2}$ & $\mathbf{0}$ \\
1. Taxa Richness (a) & $>80 \%$ & $60-80 \%$ & $40-60 \%$ & $<40 \%$ \\
2. Hilsenhoff Biotic Index (b) & $>85 \%$ & $70-85 \%$ & $50-70 \%$ & $<50 \%$ \\
3. Ratio of Scrapers/ Filt. Collectors (a, c) & $>50 \%$ & $35-50 \%$ & $20-35 \%$ & $<20 \%$ \\
4. Ratio of EPT and Chironomid Abundances (a) & $>75 \%$ & $50-75 \%$ & $25-50 \%$ & $<25 \%$ \\
5. \% Contribution of Dominant Taxon (d) & $<20 \%$ & $20-30 \%$ & $30-40 \%$ & $>40 \%$ \\
6. EPT Index (a) & $>90 \%$ & $80-90 \%$ & $70-80 \%$ & $<70 \%$ \\
7. Community Loss Index(c) & $<0.5$ & $0.5-1.5$ & $1.5-4.0$ & $>4.0$ \\
8. Ratio of Shredders/Total (a, c) & $>50 \%$ & $35-50 \%$ & $20-35 \%$ & $<20 \%$ \\
\hline
\end{tabular}

(a) Score is a ratio of study site to reference site $\mathrm{x} 100$.

(b) Score is a ratio of reference site to study site $x 100$.

(c) Determination of Functional Feeding Group is independent of taxonomic grouping.

(d) Scoring criteria evaluate actual percent contribution, not percent comparability to the reference station.

(e) Range of values obtained. A comparison to the reference station is incorporated in these indices.

Associated with each of the metrics are "critical" values. Table 1, shown above, comes directly from the EPA and depicts the metrics, the "critical" values, and also the methodology of classifying a stream. However, since there was no documentation, we determined that none of these "critical" values have statistical justification. It was decided to analyze these "critical" values in order to determine, statistically, how good they are. A slight modification was made to all of the "critical" values that did not meet the requirements of the original "critical" value. A notable discrepancy between 
the standard "critical" value and a modified "critical" value could result in a change of the "critical" value, which now has a statistical foundation.

The basic concept behind these RBP's is a quick and easy method of selecting and identifying 100 organisms from a sample to the lowest taxon possible. The main question that arises is how dependable is this type of sample in designating the health of a benthic community through various indices? More importantly, how often would two samples of 100 organisms, which are both taken from the same reference population, be considered different? The problem that is of concern is the Type I error or $\alpha$ level of a decision process. Statistically, the Type I error is rejecting the null hypothesis of equality when in fact the null hypothesis is true (McClave, Dietrich and Sincich, 1997). In this case, every time a sample is considered to be different from another from the same population, essentially, a Type I error is committed. In general, an acceptable probability for a decision process Type I error level is 0.05 .

It is generally much easier and less time consuming to identify taxa to the family level than it is to get to the genus or even the species level. Basically, a trained biologist can identify organisms to the species level, whereas, a student in biology can correctly identify organisms to the family level. One way of reducing the cost of benthic surveys could be to shorten the time needed to identify and reduce the expertise required for identification, by using a lower level of taxonomic resolution, that is, identifying specimens to higher taxonomic levels such as family or phylum. (Olsgard, Somerfield, and 
Carr, 1997). In order to evaluate this, a sample of 200 organisms identified at the family level was compared with a sample of 100 organisms identified at the genus (species) level, from the same population. Basically, a sample of 200 organisms identified at the family level can be less expensive and less time consuming, compared to a sample of 100 organisms at the genus level. Both of these provide an easier method of identifying macroinvertebrates that can be significantly cheaper and display certain characteristics of stream metrics. Using these ideas compiled with the two metrics stated above, it will be shown how reliable this method of sampling (RPB) is to characterize the overall health of a benthic community.

\section{Methodology}

As mentioned above, the RBP's give specific guidelines for each of the metrics. Together, all of the metrics helps biologists categorize the overall condition of a stream. For example, a value that is less than 0.80 or $80 \%$ of a reference site for taxa richness would indicate a degradation of that stream. Naturally, as the value increases to 1 or $100 \%$ would indicate a greater similarity of the two sites. The critical value associated with the metric, CLI, is 0.50 . The equation for CLI is as follows: $\mathrm{CLI}=(\mathrm{d}-\mathrm{a}) / \mathrm{e}$, where

$$
\begin{aligned}
& d=\text { total number of taxa present in reference site } \\
& a=\text { number of taxa common to both samples } \\
& e=\text { total number of taxa present in site of comparison }
\end{aligned}
$$

Upon observation, it can be seen that the range of values for this metric vary from zero to "infinity" and it naturally follows that as this value increases to 
infinity there is a greater difference between the reference site and the site of comparison. This trend towards "infinity" indicates a degradation of the stream and therefore values greater than 0.50 would be considered impaired.

In order to analyze these two "critical" values, a reference site and a site of comparison was needed. Specifically, we used Monte Carlo simulation to simulate sampling from each of the Vermont reference sites in our database. This database consisted of counts of species that were found by biologists at certain streams in Vermont. The species were taxonomically identified to both family and genus level. For the purpose of this paper, the first simulation on a population was deemed the reference site and the second simulation on the same population were considered to be the site of comparison. If two samples of 100 organisms, a reference and comparison site, were simulated from the same population, how many times would they be determined to be different? In order to do this the metrics, taxa richness and CLI, will be calculated from each simulated reference site and site of comparison and examined individually.

Data that was obtained from Vermont streams was the basis for creating population models using Monte Carlo simulations. These Vermont streams were sampled reference sites. All sites were considered unimpaired, thus constituting a range of reference stream populations that we used to make our simulations more realistic. These simulations naturally mimic the properties of the benthic macroinvertebrate community. The data consisted of a list of $x$ different species identified at the genus (species) level and $y$ 
different sites of streams. This created a matrix of $x$ rows and $y$ columns. For each site, a count of each species was given. A new matrix was calculated which consisted of proportions $\mathrm{p}_{1} \mathrm{p}_{2} \ldots \mathrm{p}_{\mathrm{k}}$ where $\mathrm{k}$ is the number of species that had a count greater than zero. For each y stream, a 100 by 100 matrix was simulated at random using the associated proportions. This simulation matrix represented a sample size of $n=100$ organisms versus a $\mathrm{z}=100$ repetitions of the counts per species. For each population model, two simulation matrices were generated, one for a reference site and another for a site of comparison. For both of the sites in all y populations, the taxa richness and CLI values were calculated. In actuality, there were 100 values for taxa richness and 100 values for CLI. Finally, each of the 100 taxa richness values were compared to the "critical" value associated with it and accordingly for CLI. The number of times that the obtained value violated the "critical" value was divided by 100 and recorded. This value now signifies the probability of Type I error associated with each metric. Traditional acceptable level for the probability of a Type I error is 0.05 .

In addition to recording the estimates of Type I error rates for taxa richness and CLI, we also calculated the "true" richness and evenness for each Vermont sampled community. These two values were picked because they represent the two measurements that make up the concept of species diversity that is widely acknowledged by biologists. Taxa richness was easily computed by simply counting the number of different species in each of the stream samples. For many decades field ecologists had known that most communities 
of plants and animals contain a few dominant species and many species that are relatively uncommon. Evenness measures attempt to quantify this unequal representation against a hypothetical community in which all species are equally common (Krebs, 1999). Basically, evenness is a measure of how evenly spread all of the species in a community are. There are several different methods of calculating evenness measures, but the Shannon-Wiener function was most suitable. Strictly speaking, the Shannon-Wiener measure of information content should be used only on random samples drawn from a large community in which the total number of species is known (Pielou 1966). The Shannon-Wiener function is as follows:

$$
H^{\prime}=-\sum_{i=1}^{s}\left(p_{i}\right)(\log x p i)
$$

where

$$
\mathrm{H}^{\prime}=\text { index of evenness }
$$

$\mathrm{p}_{\mathrm{i}}=$ proportion of total sample belonging to $i$ th species

$$
\mathrm{s}=\text { number of species }
$$

The $\log _{\mathrm{x}}$ function represents that any base of logarithms can be used, since they are all convertible to one another by a constant multiplier. The Shannon-Wiener measure increases with the number of species in the community and in theory can reach very large values. The theoretical maximum value is $\log (\mathbf{S})$ and the minimum value as $N$ approaches $S$ is $\log$ $[N /(N-S)]$ where $N$ is the number of individuals in the community (Fager 1972). Since the Shannon-Wiener measure can range from 0 to $\infty$, it was 
standardized to a 0 to 1 scale by dividing $\mathrm{H}^{\prime}$ by $\log (\mathrm{S})$, where $\mathrm{S}$ is the total number of species (taxa richness).

The next change that was made consisted of altering the sample size. A sample size of 100 is recommended by the EPA for the RPB protocols. An increase in sample size could actually augment the accuracy of the sample taken. It was noted that if Type I error was less than 0.05 in a sample size of 100 , an increase in sample size would also result in a Type I error less than 0.05. If Type I error was greater than 0.05 in a sample of 100 , there becomes a better chance that an increase in sample size could reduce the chances of making a Type I error. An increase to a sample size of 200 was made to all of the stream sites that had failed to be below the required 0.05 value. We also examined the possibility of increasing the sample size to 300 in some cases to ensure that we obtained results that were statistically acceptable. Doberstein, Karr, and Conquest (2000) developed an experiment using 100, 200, 300, 500, 700 , and 1000 simulated organisms to determine the best sample size for certain metrics.

It is known that identifying species to the genus (species) level can be a rather tedious job. In fact, as stated above, a trained biologist is generally required. Identification of species to the family level is much easier as well as less time consuming. The next part of this research consisted of Monte Carlo simulations done on organisms identified to the family level. A sample of 100 organisms identified at the family level would be much more cost effective than a sample of 100 organisms identified at the genus (species) level. First of 
all, in order to be able to compare family and genus levels, it is essential that each of the individual stream sites be exactly the same. For example, the organisms identified at the family and genus level must both come from the same site and actually from the same sample. However, the streams from the family level were not all the same as the streams from the genus level, so the streams that were common to both were used for comparisons. All of the previous information that was gathered for the genus level observations was similarly gathered for the family level. After finishing the 100-organism sample for the family level, we then examined the possibility of obtaining a 200-organism sample at the family level as well as a 300-organism sample.

The final alteration that was made consisted of now changing the "critical" value that was given by the RBP. These two values for taxa richness and CLI were 0.80 and 0.50 respectively. Because there is no known statistically valid reason for these values, it was decided to analyze exactly how accurate these values are and examine to see if a slight variation to them is needed. For this purpose, the 100 -organism sample from the genus (species) level was used to analyze this change in "critical" levels.

\section{Simulation}

The population models used in this study were generated using Monte Carlo simulations. Monte Carlo methods are stochastic techniques - meaning they are based on the use of random numbers to investigate problems (Woller 1996). Using a random number generator from Excel species were "simulated" 
and recorded based on the computed proportions from the raw data. For each Vermont stream we knew a vector of species proportions $[\mathrm{p} 1, \mathrm{p} 2, \ldots, \mathrm{pk}]$.

Using this as a multinomial probability model, we simulated counts of species.

This simulation is equivalent to sampling organisms with replacement from existing stream samples as done by Doberstein, Karr, and Conquest (2000).

One completion of simulations on a single stream population consisted of generating either two 100 (samples) by 100 (repetitions) matrices, two 200 (samples) by 100 (repetitions) matrices or two 300 (samples) by 100(repetitions) matrices of random samples of organisms. There were a total of 38 stream sites for the genus level and a total of 40 sites for the family level. All of the streams were used for the 100 sample simulations for both genus and family level. There were a total of 28 streams that were common to both the genus data and the family data. These streams were used in all of the comparisons as mentioned above.

\section{Results}

\section{A: Sample size of 100 at the Genus Level}

All of the descriptions in this section represent sites in which we simulated a sample size of 100 organisms from the Vermont streams. The data from these streams were all identified to the genus level. Figure 1 depicts a graph of the "true" taxa richness values plotted against the alpha level for richness. As stated above, alpha level or Type I error, is the probability of saying the sites are different when in fact the sites are the same. The associated "critical" value for richness is 0.80 as stated above. There are 15 
sites out of the 38 different sites that fall above the 0.05 alpha level and 2 that are considered borderline since they are exactly 0.05 . This is a high number of failures at $44.74 \%$ and is generally unacceptable. Upon looking at the graph, there does not seem to be any kind of trend that is associated with either high or low richness values. Figure 2 represents the "true" taxa richness values plotted against the alpha level for CLI. The critical value is 0.50 for CLI. Observe that there are 7 sites out of 38 sites that fall above the 0.05 alpha level and 2 that have alpha values that are exactly 0.05 for a total of 9 out of 38 (23.68\%). This is still a high rate of failure but somewhat better. There does not seem to be any type of trend associated with this graph either. Figure 3 shows the graph of the "true" evenness values against the alpha values for taxa richness. Since the graph is plotted with the same alpha values, we have the same number of failures as in Figure 1. There seems to be some kind of indication that the higher evenness values corresponding to lower alpha levels and lower evenness values having higher alpha levels. However, eliminating the 3 outliers with low evenness values could eliminate the possibility of any trend. Figure 4 represents the "true" evenness values graphed against the alpha levels for CLI. Because the graph utilizes the same alpha values, there is the same number of failures as in Figure 2. Similar to Figure 3, there seems to be evidence of higher evenness values having lower alpha levels. This trend appears to be somewhat stronger than in Figure 3. If the same 3 outliers are removed, an argument can still be made that there still is a trend. Figure 5 reveals the graph of actual richness vs. actual evenness of each site. As we can 
see, there is no specific richness and evenness combination that would suggest either high or low alpha levels. Figure 6 is the same type of graph as Figure 5 except we are using the CLI "critical" values instead of taxa richness. As in Figure 5, there does not appear to be any high or low richness and evenness values that have alpha levels that are similar.

\section{B: Sample size of 200 at the Genus Level}

As stated above all of the sites in this category were the sites that had alpha levels that were at or exceeded 0.05 . Therefore there are 17 for the richness alpha values and 9 for the CLI alpha values. Figure 7 shows the graph of "true" richness versus alpha level for richness. We can see that now out of the 17 that failed before, only 2 sites exceeded the alpha level 0.05 and 2 others were exactly at 0.05 . Assuming that all of the sites in the sample size of 100 that were below 0.05 level would also have been below 0.05 in the sample size of 200 , we would now have a total of only 4 out of 38 that were at or exceeded the 0.05 level. This is about $10.5 \%$, which is much more acceptable statistically. It is noted that by changing the sample size from 100 to 200 , there is a substantial improvement that has occurred. We have gone from $44.74 \%$, at sample size of 100 , equal to or above the 0.05 alpha level to $10.5 \%$, at the sample size of 200. Upon observing Figure 7, we can see that there is generally no trend. However, by removing the point $(36,0,08)$ one could detect some kind of rising trend from left to right, but this is a very weak trend at best. Figure 8 represents the graph of "true" richness versus the alpha level for CLI. Immediately we can see that there are no values that are above the 
0.05 alpha value. This means that none of the sites, including the one's which had alpha values below 0.05 for the sample size of 100 , had high alpha values associated with the CLI values. Figure 9 displays the graph of "true" evenness versus the alpha level for richness. As in Figure 7, there are 2 above the 0.05 alpha level and 2 that are exactly 0.05 which is much better than the sample size of 100 . The biggest feature of this graph is the somewhat apparent trend occurring. There is a downward slope from left to right. This suggests that the sites with higher failure rates are associated with lower evenness values. Figure 10 shows graph of "true" evenness versus the alpha level for CLI. As in Figure 8, there are no alpha values above 0.05 , therefore an attempt to discover any kind of trend is unnecessary. Figure 11 depicts the diagram of "true" richness values plotted against the "true" evenness values with the alpha levels for richness as the "third dimension", as in Figure 5. Since there are only four values that either exceeded or were exactly 0.05 , it is nearly impossible to definitively state that there is any trend at all. Finally, Figure 12 displays the graph of "true" richness vs. "true" evenness, with the alpha level for CLI as the "third dimension". As in Figure 8 and Figure 10, there are no values that exceed the 0.05 alpha level therefore it is unnecessary to determine if any trend is apparent.

\section{C: Sample size of $\mathbf{3 0 0}$ at the Genus Level}

This section consists of all of the sites from Section B that had alpha values that still exceeded the 0.05 level for richness only. Another increase in sample size to 300 would help to reduce the number of sites with alpha values 
greater than 0.05 . From Section B there were still 4 out of $38(10.5 \%)$ sites for richness that qualified for this increase in sample size. Since there are only 4 points, any type of graph would be unnecessary. Of the 4 sites, there was still one site that again had alpha values at or above 0.05 . Even though we have increased the sample size from 200 to 300 , we have only one site out of 38 (2.6\%) that had an alpha value at or above 0.05 . This is a low percentage rate however the sample size is a rather large increase from the original sample size of 100 .

\section{D: Sample size of $\mathbf{1 0 0}$ at the Family Level}

For all of the graphs in this section, there are a total of 40 sites that were examined, as stated above, and the richness and CLI "critical" values are the same, at 0.80 and 0.50 respectively. Figure 13 represents the graph of "true" richness versus the alpha level for richness. As we can see, there are 30 sites that exceed the 0.05 alpha level and one, which is exactly at 0.05 . With a percentage of $77.5 \%$, this means that 3 out of every 4 sites failed to be below the alpha level of 0.05 , with a good portion of them even exceeding the 0.10 alpha level. There does not appear to be any trend associated with "true" richness values, since, all of the data seems to be scattered around the middle. Figure 14 represents the graph of "true" richness values versus the alpha level for CLI. Here there are only three sites that have the alpha value greater than 0.05 and one that is at 0.05 exactly. With 4 out of $40(10 \%)$ exceeding the 0.05 alpha level for CLI, this generates an interesting thought. From the same data, we get two entirely different results. On one hand, the richness values are 
statistically very poor and on the other, the CLI values are much more acceptable. Figure 15 depicts the graph of "true" evenness versus the alpha level for richness. Looking at Figure 15, we observe that again there are a total of 31 sites that exceed or are exactly 0.05 , for reasons stated above. There is an interesting observation, however, we notice that occurs in Figure 15. For values of "true" evenness up to 0.70 , there are no sites that have alpha levels that are less than 0.05 . In other words, all of the sites that had "true" evenness values less than 0.70 had an alpha level above 0.05 . Figure 16 shows the relationship of the "true" evenness versus the alpha level for CLI. As in Figure 14 , there are only a total of four sites that are at or above the 0.05 alpha level. Observing Figure 16, a downward slope can be identified. Upon looking at the values that are less than 0.70 for "true" evenness, we can see that there is only one site that has an alpha level of 0.00 and all of the higher alpha levels occur below this "true" evenness value. In addition, there are 11 sites that have alpha levels of 0.00 and are above 0.70 . Figure 17 graphs the "true" richness values versus the "true" evenness values in which symbols are given to a specific range of alpha levels for richness as in Figure 5. Looking at the sites that had alpha levels less than 0.05 , we can observe that all of them have evenness values greater than 0.70 , however, there is no relationship between both richness and evenness in accordance with alpha levels. Finally, Figure 18 reveals the "true" richness versus the "true" evenness values with symbols representing a specific range of alpha levels for CLI, as in Figure 6. Since there are only four observations that are above the alpha level of 0.05 , it is 
difficult to detect any type of trend associated with high alpha levels.

However, there are two notes to make: 1) All four of the sites have richness values between 20 and 26 , evenness values below 0.65 . 2) The majority of the sites with alpha levels below 0.05 have evenness values above 0.65 and vice versa.

\section{E: Sample size of 200 at the Family Level}

Since the results of Section D show poor statistical behavior for a sample size of 100 at the family level, we now look at increasing the sample size to 200 for the family level taxonomic identification. All of the sites in this section are the ones that were not below the alpha level of 0.05 , as mention above. Again, there were 31 sites that "qualified" for the richness and only 4 for CLI. Since there are only 4 for CLI, there are no graphs to represent the data because no observations can be made with so little data. Figure 19 represents the "true" richness versus the alpha level for richness. We can observe that there are 9 sites that have alpha levels greater than 0.05 and 2 that have alpha levels of 0.05 exactly. If we assume that the sites that had alpha levels less than 0.05 at a sample size of 100 also have alpha levels less than 0.05 at a sample size of 200 . We have a total of 11 out of $40(27.5 \%)$ sites that "fail" to be below 0.05 alpha level. Referring to Figure 19 again, it is apparent that there is no specific pattern associated with the richness values. Next, Figure 20 displays the "true" evenness versus alpha levels for richness. As in Figure 19, there are now only 11 "failure" sites. Looking at only the sites with alpha levels greater than 0.05 , we observe that there is an apparent downward 
trend. Figure 21 shows the graph of "true" richness values versus "true" evenness values, as in Figure 5. Other than the fact that there is a relationship between both low richness and evenness values and high richness and evenness values, there is no observable trend associated with the alpha levels included. For the 4 sites connected with alpha levels greater than 0.05 for CLI, there was actually one that still was exactly at the 0.05 alpha level. The values went from 0.10 to $0.05,0.09$ to $0.01,0.06$ to 0.01 and 0.05 to 0.02 . By comparing the results from the 100 -sample size at the genus level and the 200 -sample size at the family level, we observe that the richness alpha levels for the 200 sample size at the family level are much better than the 100 -sample size at the genus level. For richness in the 100 -sample size at the genus level we had 17 out of $38(44.74 \%)$ that failed to be below the 0.05 alpha level. For richness in the 200 -sample size at the family level we had 11 out of $40(27.5 \%)$ that had values at or above the 0.05 alpha level. This suggests that for richness, a 200sample size at the family level is much better statistically than a 100 -sample size at the genus level. Comparing the 100-sample size at the genus level and the 200 -sample size at the family level for CLI, we can see that the 200 -sample size at the family level are much better than the 100 -sample size at the genus level. We obtained 9 out of $38(23.68 \%)$ for the 100 -sample size at the genus level and 1 out of $40(2.5 \%)$ for the 200 -sample size at the family level. This also suggests that for CLI, a 200-sample size at the family level is much better statistically than a 100 -sample size at the genus level. 


\section{F: $\quad$ Sample size of 300 at the Family Level}

Since there were still a rather large number of sites that had alpha values at or above 0.05 for richness only, we decided to increase the sample size again from 200 to 300 . This would provide us with fewer sites with alpha values at or above 0.05 . From Section E we still had 11 out of 40 (27.5\%) sites for richness that "qualified" for this section. Figure 22 shows the graph of "true" richness versus the alpha value for richness and Figure 23 displays the graph of "true" evenness versus the alpha values for richness. We can observe from Figure 22 or 23 that there are 3 out of the 11 sites that still have alpha values at or above 0.05 . Taking the results from Section $\mathrm{D}$ and $\mathrm{E}$, this gives us only 3 out of $40(7.5 \%)$ sites that still have alpha values at or above 0.05 after a sample size of 300 .

\section{G: Sample size of 100 at the Genus level ("critical" value change)}

An alternative to changing the sample size and/or identification level would be to look at and possibly adjust the "critical" levels for richness and CLI. Upon examining the data for taxa richness, it was discovered that we wanted a less strict "critical" value since we wanted to reduce the chances of rejection. In order to obtain this, the data had to be carefully observed prior to judgment. A lower value would decrease the chances for a Type I rejection and therefore help to not make a wrong decision. It was decided that the change to the "critical" value would be from 0.80 to 0.75 for richness. We would reduce the "critical" value for richness by 0.05 until there were an acceptable percentage of alpha values. The change for CLI was from 0.50 to 
0.55. The "critical" value for CLI would increase by 0.05 until an acceptable percentage of alpha values was found. Since we are only changing the "critical" value, it is not necessary to discuss trends since the data are not changing at all. Any type of trend discovered before would also hold true even after changing the "critical" value. For the sake of completion there are graphs for both "true" richness and "true" evenness. Figures 24 and 25 represent the graph of "true" richness and "true" evenness versus alpha level for richness with the "critical" value of 0.75 , respectively. It is apparent from Figure 24 and Figure 25 that there are only 2 out of $38(5.3 \%)$ sites that have alpha levels greater than 0.05 . There is a substantial difference between the 100 -sample size at the genus level with no "critical" value change and the 100-sample size at the genus level with a change from 0.80 to 0.75 . With no change in "critical" value for richness, there are 17 out of $38(44.74 \%)$ sites above the 0.05 alpha level and with the change there are only 2 out of $38(5.3 \%)$ sites. Therefore there is significant statistical evidence that changing the rule has a very beneficial influence. Finally, Figures 26 and 27 depict the "true" richness and "true" evenness versus the alpha levels for CLI with a "critical" value of 0.55 , respectively. We can see that there is one site that has a value greater than the 0.05 alpha level and 3 that are equal to 0.05 . There were 4 out of 38 (10.5\%) sites with the change in CLI "critical" value and 9 out of 38 (23.68\%) sites from the 100-sample size at the genus level with no change in "critical" value. From this, we observe that there are better results when there is a change in CLI from 0.50 to 0.55 . Since there were still 9 out of 38 sites with the 
change from 0.50 to 0.55 , we increased the "critical" value to 0.60 . Figure 28 and Figure 29 represent the graphs of "true" richness and "true" evenness versus alpha level for CLI with the "critical" value of 0.60 , respectively. We can observe from Figure 28 or 29 that there are no sites that exceed the 0.05 alpha value. Since there are no sites that have alpha values above 0.05 , the "critical" value of 0.60 was considered the ending point for richness.

\section{H: Sample size of 100 at the Family level ("critical" value change)}

We also decided to change the "critical" value for the family level in addition to the change from Section G. The change for richness was the same as in Section E, from 0.80 to 0.75 and decreasing by 0.05 until an acceptable percentage of alpha values is reached. The change for CLI was also the same as in Section E. Figure 30 and Figure 31 show the graphs of "true" richness and "true" evenness versus alpha level for richness with the "critical" value of 0.75 , respectively. We can observe from both Figure 30 and 31 that there are 8 sites that have alpha levels above 0.05 and 2 that have alpha levels at 0.05 exactly. If we compare the results from the 100 -sample size at the family level without a "critical" value change, we can observe a significant decrease in the number of sites that have alpha levels above 0.05 . By changing the "critical" value from 0.80 to 0.75 , the number drops from 31 out of $40(77.5 \%)$ sites that had alpha levels above 0.05 with no change, to 9 out of $40(22.5 \%)$ sites that had alpha levels above 0.05 with the change from 0.80 to 0.75 . It is obvious that there is a distinct difference between the two "critical" values and it is apparent that the "critical" value of 0.75 is statistically more valid and accurate 
than the 0.80 value. The next step was to change the "critical" value for richness from 0.75 to 0.70 . This would prove to improve the results even further than the change from 0.80 to 0.75 . Figures 32 and 33 display the "true" richness and "true" evenness versus the alpha values for richness with the "critical" value of 0.70 , respectively. Observing either Figure 32 or 33, we can see that there are 2 sites that have alpha values above 0.05 and one site that has an alpha value exactly at 0.05 . By changing the "critical" value from 0.75 to 0.70 we now have only 3 out of $40(7.5 \%)$ sites that have alpha values above 0.05 . This is a good percentage without changing the "critical" value too much. Figures 34 and 35 represent the graphs for "true" richness and "true" evenness versus the alpha values for CLI with the "critical" value of 0.55 , respectively. Upon observing Figure 34 and 35, it is apparent that there are 2 sites that have alpha levels greater than 0.05 and one that is 0.05 exactly. In comparison of the data obtained from the 100 -sample size at the family level with no "critical" value change and the 100 -sample size at the family level with a change, we can see that again there is a small increase between the two. Now we have values that go from 4 out of $40(10 \%)$ sites with no "critical" value change to 3 out of 40 (7.5\%) sites with the change from 0.50 to 0.55 . There is not much of a drop in the number of sites with alpha levels above 0.05 , however we are getting better results. In order to make this, 3 out of 40 ( $7.5 \%)$ sites better, we increased the "critical" value again, from 0.55 to 0.60 . Figure 36 and Figure 37 represent the graphs for "true" richness and "true" evenness versus the alpha level for CLI with the "critical" value of 0.60 , 
respectively. Upon observing either of the two figures, we observe that there is one site that had an alpha value greater than 0.05 . Figure 36 has 15 sites that have the same values for more than one site. By changing the "critical" value from 0.55 to 0.60 , we have decreased the number of sites with alpha values above 0.05 . This number went from 3 out of $40(7.5 \%)$ with the "critical" value of 0.55 , to 1 out of $40(2.5 \%)$ with the "critical" value of 0.60 .

\section{Discussion}

\section{A: The effects of increasing the sample size}

For this analysis, there were three different factors, sample size, taxonomic level and "critical" value, which were applied to two metrics, richness and CLI, separately. It is noted that we are not comparing richness versus CLI for any of these results. First of all, from the original criteria for the RBP's, i.e. sample size of 100 at the genus level we observed a Type I error rate of $44.74 \%$ for the richness metric and a $23.68 \%$ rate for the CLI metric. Initially, we are changing the sample size while holding the taxonomic level and "critical" value constant. As we can see from Table 2 (shown below), increasing the sample size for richness at the genus level with the "critical" value of 0.80 , there was a substantial decrease in the percentage of sites with alpha values greater than 0.05 . At a sample size of 300 , only a $2.6 \%$ of sites had an alpha level greater than 0.05 . Looking again at Table 2 (shown below), now at the family level, we can see that the percentage began at $77.5 \%$ with a sample size of 100 , and declined to $7.5 \%$ at a sample size of 300 . This $7.5 \%$ is still good however with a sample size of 300 , we had hoped of getting a 
smaller percentage. Increasing the sample size to 400 would have been a rather high increase in sample size for a relatively low decrease in percentage level.

\section{Table 2.}

\section{Summary Totals for Richness}

Taxonomic Level Sample Size Rule \% of Sites

\section{Exceeding 0.05}

\begin{tabular}{llll}
\hline Genus & 100 & 0.80 & $44.74 \%$ \\
Genus & 200 & 0.80 & $10.5 \%$ \\
Genus & 300 & 0.80 & $2.6 \%$ \\
Family & 100 & 0.80 & $77.5 \%$ \\
Family & 200 & 0.80 & $27.5 \%$ \\
Family & 300 & 0.80 & $7.5 \%$ \\
Genus & 100 & 0.75 & $5.3 \%$ \\
Family & 100 & 0.75 & $22.5 \%$ \\
Family & 100 & 0.70 & $7.5 \%$
\end{tabular}

Referring to Table 3 (shown below), we are now looking at the CLI metric instead of richness. With the taxonomic level at genus and the "critical" value of 0.50 , we can observe that at a sample size of 200 , we have a $0.0 \%$ of sites with alpha values exceeding 0.05 . This means that we would have correctly categorized all of the sample streams in comparison with the reference streams. This is idealistically what was desired with a relatively low sample size as in this case. Changing to the family level, we see that at the sample size of 200 , we have a $2.5 \%$ of sites with alpha levels that exceed 0.05 . 
Again, we would like this number to be $0.0 \%$, however increasing the sample size to 300 would be a high increase in sample size for a relatively low decrease in percentage of alpha values above 0.05 . Changing the taxonomic level for CLI does not have as much as an effect as it for richness.

Table 3.

Summary Totals for CLI

Taxonomic Level Sample Size Rule \% of Sites

\section{Exceeding 0.05}

\begin{tabular}{llll}
\hline Genus & 100 & 0.50 & $23.68 \%$ \\
Genus & 200 & 0.50 & $0.0 \%$ \\
Family & 100 & 0.50 & $10.0 \%$ \\
Family & 200 & 0.50 & $2.5 \%$ \\
Genus & 100 & 0.55 & $10.5 \%$ \\
Genus & 100 & 0.60 & $0.0 \%$ \\
Family & 100 & 0.55 & $7.5 \%$ \\
Family & 100 & 0.60 & $2.5 \%$
\end{tabular}

\section{B: The effects of changing the taxonomic level}

For this section, we are now comparing the two different taxonomic levels while holding the sample size and the "critical" values constant, for both richness and CLI. Observing Table 2, we can see that if we compare the genus level with the family level with the same sample size and the same "critical" value, we see that our best results occur with the genus level with a sample size of 300 and "critical" value of 0.80 . For this we get a $2.6 \%$ of sites with alpha values exceeding 0.05 compared with $7.5 \%$ for the family level. We also 
observe that through all of the comparisons, the genus level yields better results. Now changing to Table 3 for CLI, we hold the "critical" value and the sample size the same and compare the genus level with the family level. By doing this, it is obvious that the genus level with a sample size of 200 and "critical" value of 0.50 gives the best results of $0.0 \%$. However, the family level with a sample size of 200 and "critical" value of 0.50 also gives excellent results with a $2.5 \%$ of alpha levels above 0.05 .

\section{C: The effects of changing the "critical" value}

This section deals with changing the "critical" value while holding the sample size and the taxonomic levels constant. Again, it is obvious that a change in the right direction will certainly decrease the percentage of sites with alpha values greater than 0.05 . However, it is noted that a deviation that is too far from the original "critical" value may be found to be unacceptable. We were looking for a low percentage of alpha values greater than 0.05 while also not straying too far from the established "critical" value. First of all, we look at the richness metric from Table 2. Looking at the genus level with a sample size of 100 and comparing the "critical" value of 0.80 with the "critical" value of 0.75 , there is a significant drop from $44.74 \%$ to $5.3 \%$. This was determined to be a good value that also did not deviate from the original "critical" value of

0.80. Moving on to the family level, we can see that changing the "critical" value from 0.80 to 0.70 yielded a $7.5 \%$ of sites with alpha values greater than 0.05 . This number is statistically good, however since the difference in "critical" value is already at 0.10 , we decided that any other change away from 
the established might be considered unacceptable. Changing to Table 3 and CLI, we now have the established "critical" value of 0.50 . At the genus level, we observe that the new "critical" value of 0.60 gives a $0.0 \%$ of alpha values above 0.05 . This is good because we did not deviate from the original "critical" value and obtained a $0.0 \%$. At the family level, we obtained a $2.5 \%$ at the "critical" value of 0.60 . For this, we could have changed the "critical" value more, but this could have been too far from the original "critical" value. It is noted that changing the "critical" value is not an easy task like changing the sample size. These values were established by the EPA and although statistically these numbers suggest that the both of the "critical" values need to be changed there is much more involvement than the scope of this research.

\section{D: Recommendations}

The results of this simulation study suggest that for the richness metric, the most efficient method of obtaining the lowest percentage of sites with alpha values exceeding 0.05 would be to change the "critical" value from 0.80 to 0.75 at the genus level with a sample size of 100 . This may not be the easiest idea to accomplish since the EPA has already established these "critical" values. However, this is a good indicator that there might need to be further investigation into changing these values.

Originally, we wanted to compare the sample of 100 at the genus level with the sample of 200 at the family level. It is shown that the sample of 200 at the family level had a lower percentage of sites with alpha levels exceeding 0.05 . However, for 
richness, the results for the sample of 300 for family level give better results than the sample of 200 for the genus level.

The most effective method of obtaining the lowest percentage of sites with alpha levels exceeding 0.05 for the CLI metric would be at the genus level with a sample size of 200 and no "critical" value change. Overall, the results for the CLI metric were much better statistically since the percentages of sites with alpha levels exceeding 0.05 were lower than for the richness metric. Looking again at the sample of 100 at the genus level versus the sample of 200 at the family level, we observe much better results for the sample of 200 at the family level. In general, it appears that all results for the sample size of 200 for family are better than the sample of 100 at the genus level, especially for richness.

The two most efficient results are only noted here because they are considered the best. However, there are other considerations that may need to be addressed on a case-by-case experiment. These include cost efficiency, time efficiency, and level of expertise among others. Each may or may not have a more important role depending on the specified conditions. Therefore it is up to the reader to determine which of the results best suits his/her experiment. 
Figure 1 - Genus Level: True Richness vs. Alpha(Richness)

Sample size $=100$

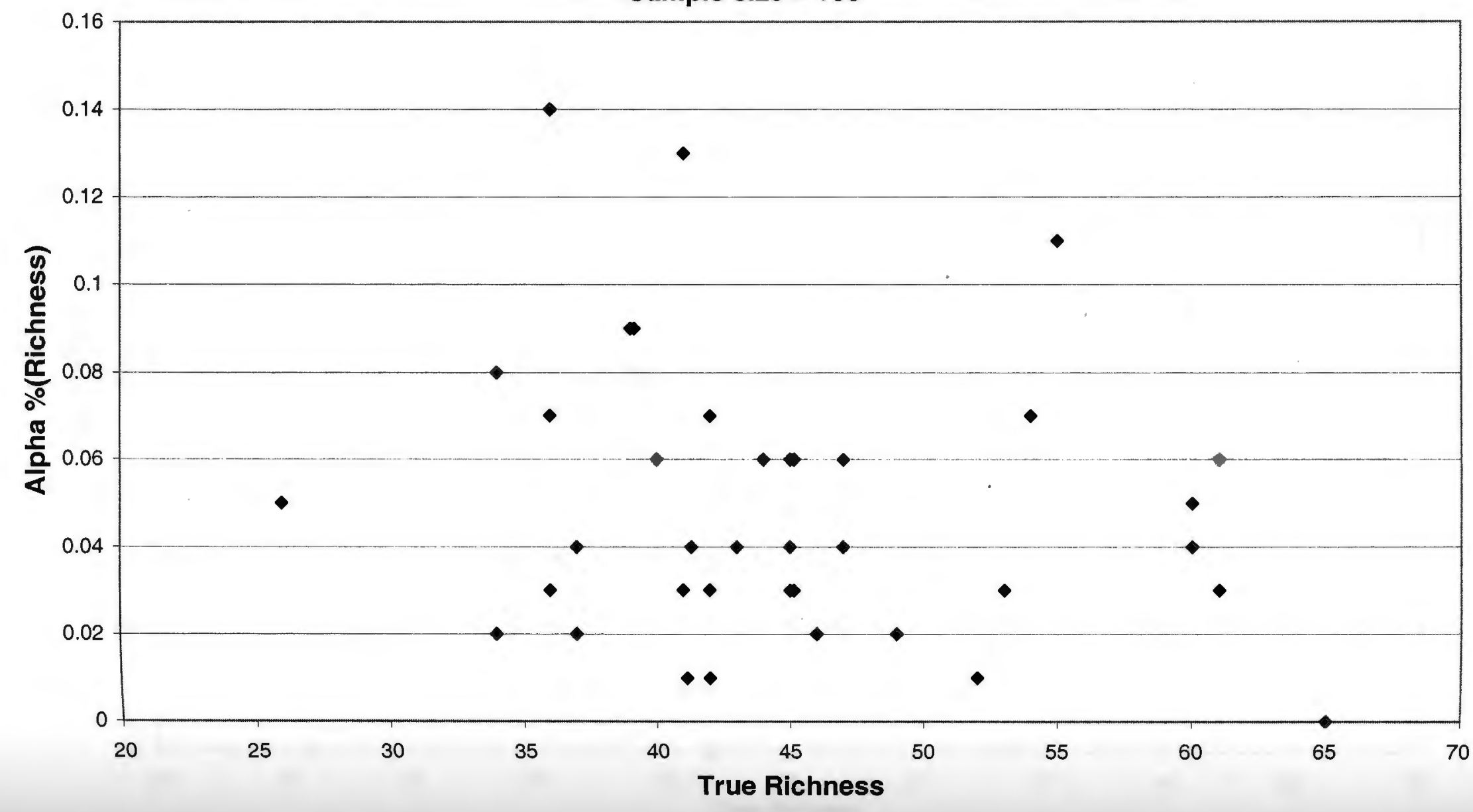


Figure 2 - Genus Level: True Richness vs. Alpha(CLI)

Sample size $=100$

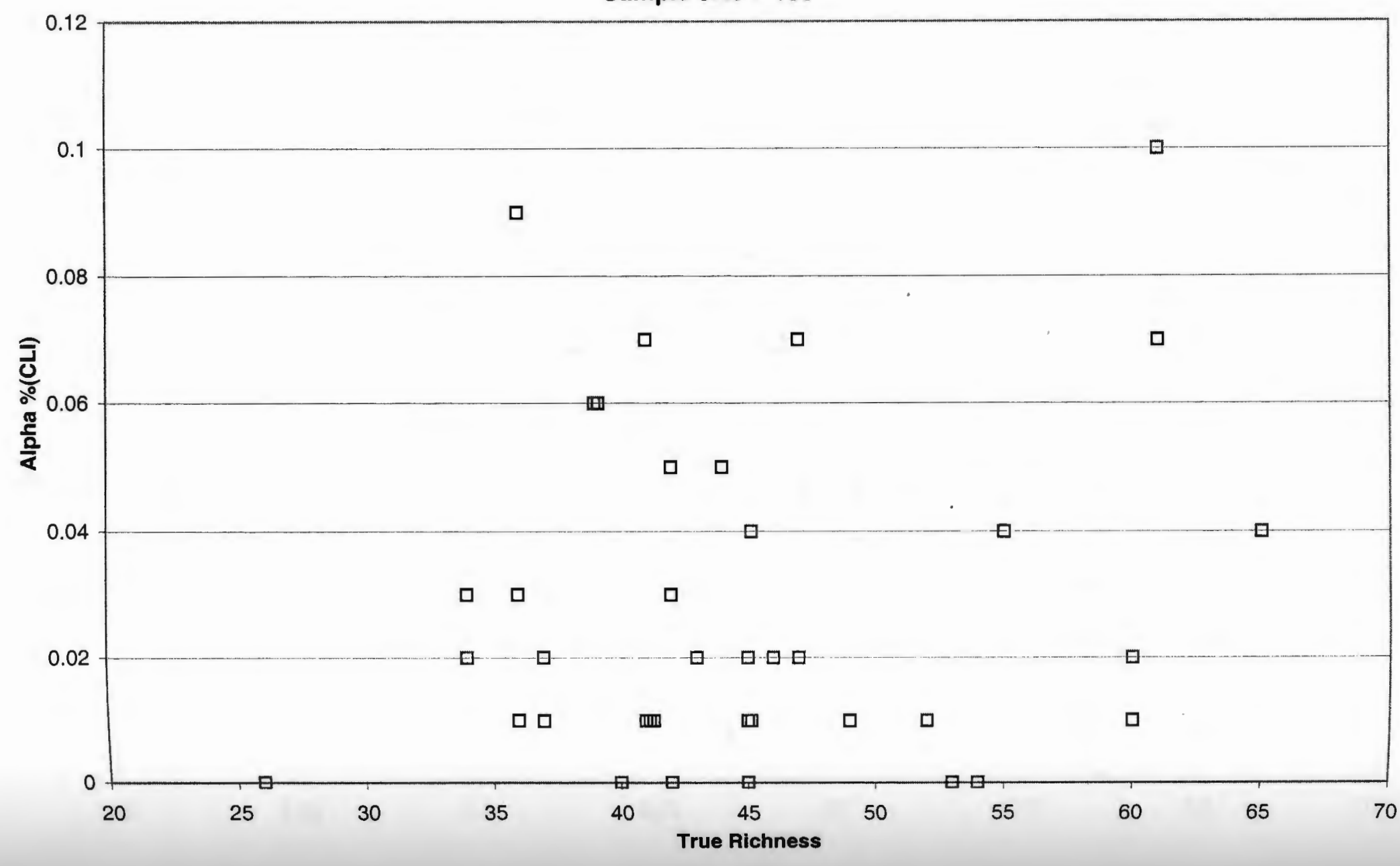


Figure 3 - Genus Level: True Evenness vs. Alpha(Richness)

Sample size $=100$

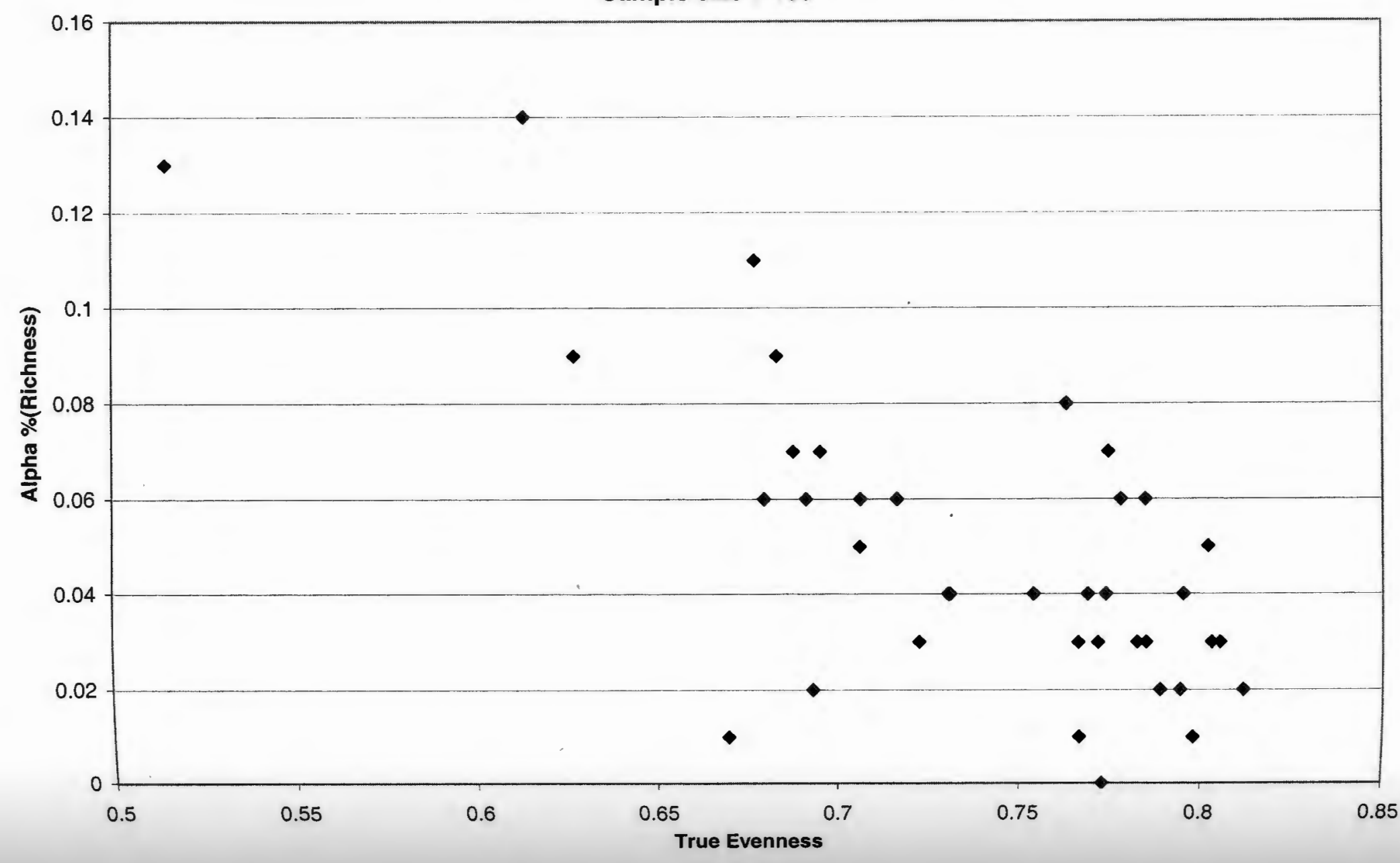




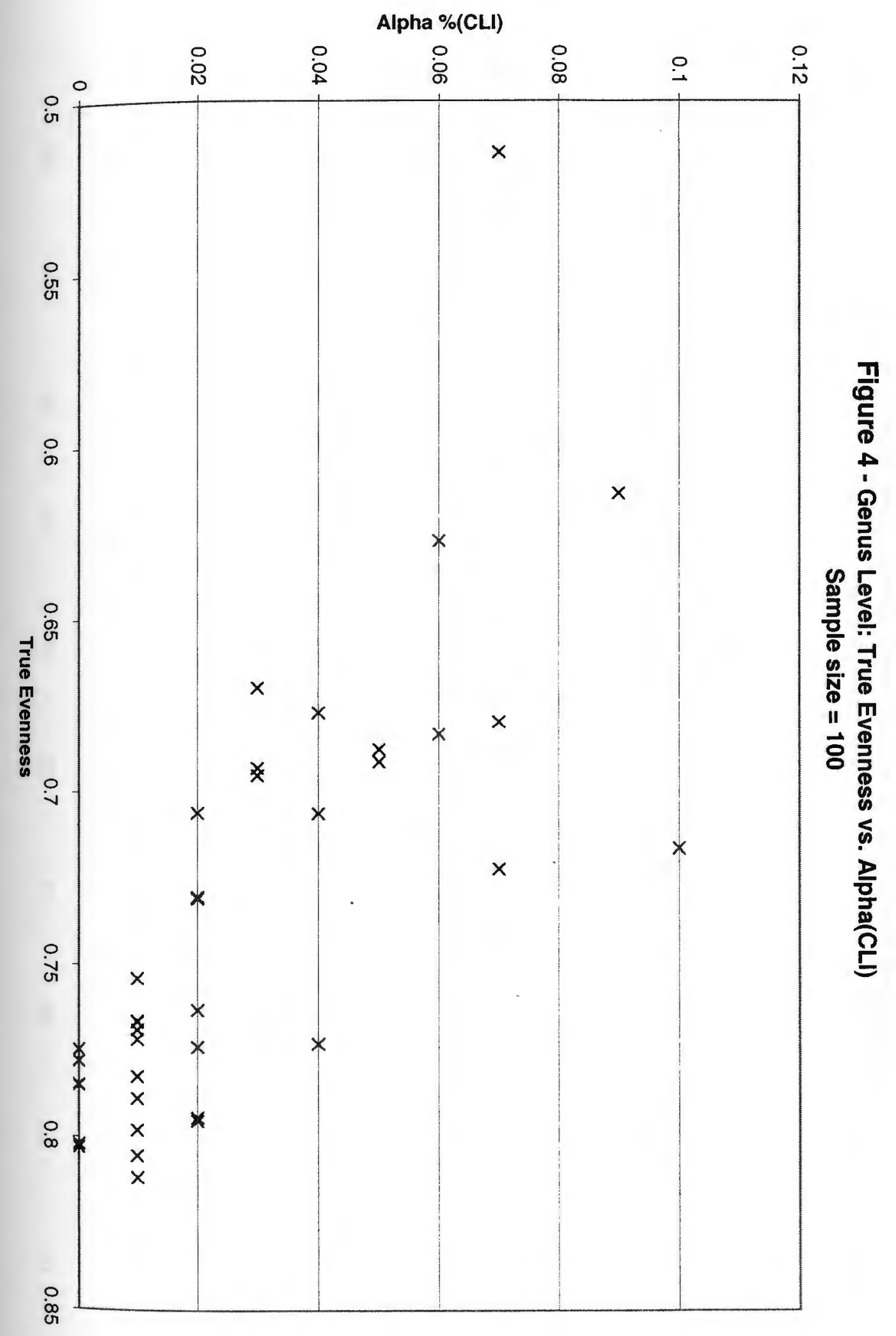


True Evenness

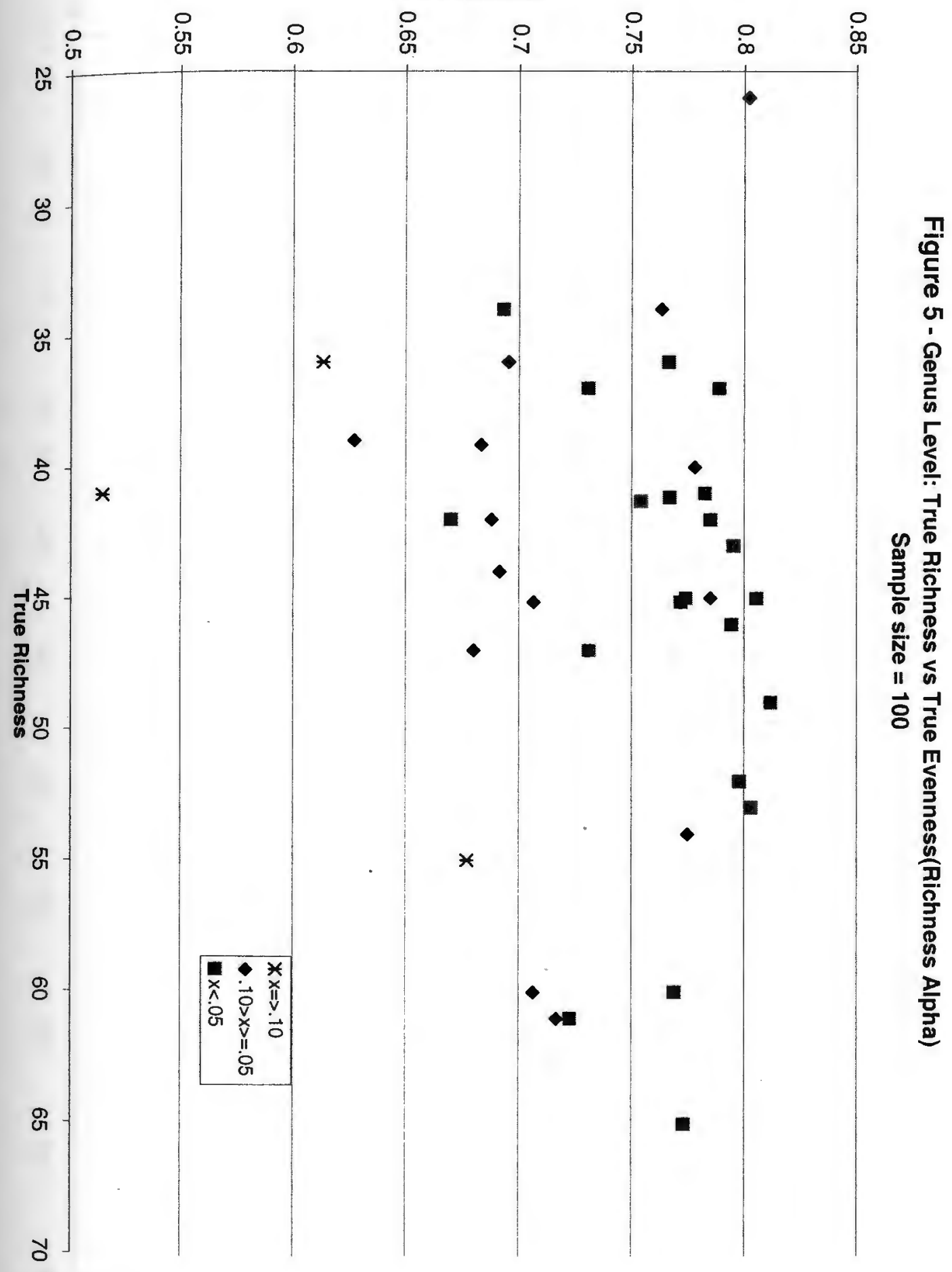


Figure 6 - Genus level: True Richness vs. True Evenness (CLI Alpha)

Sample size $=100$

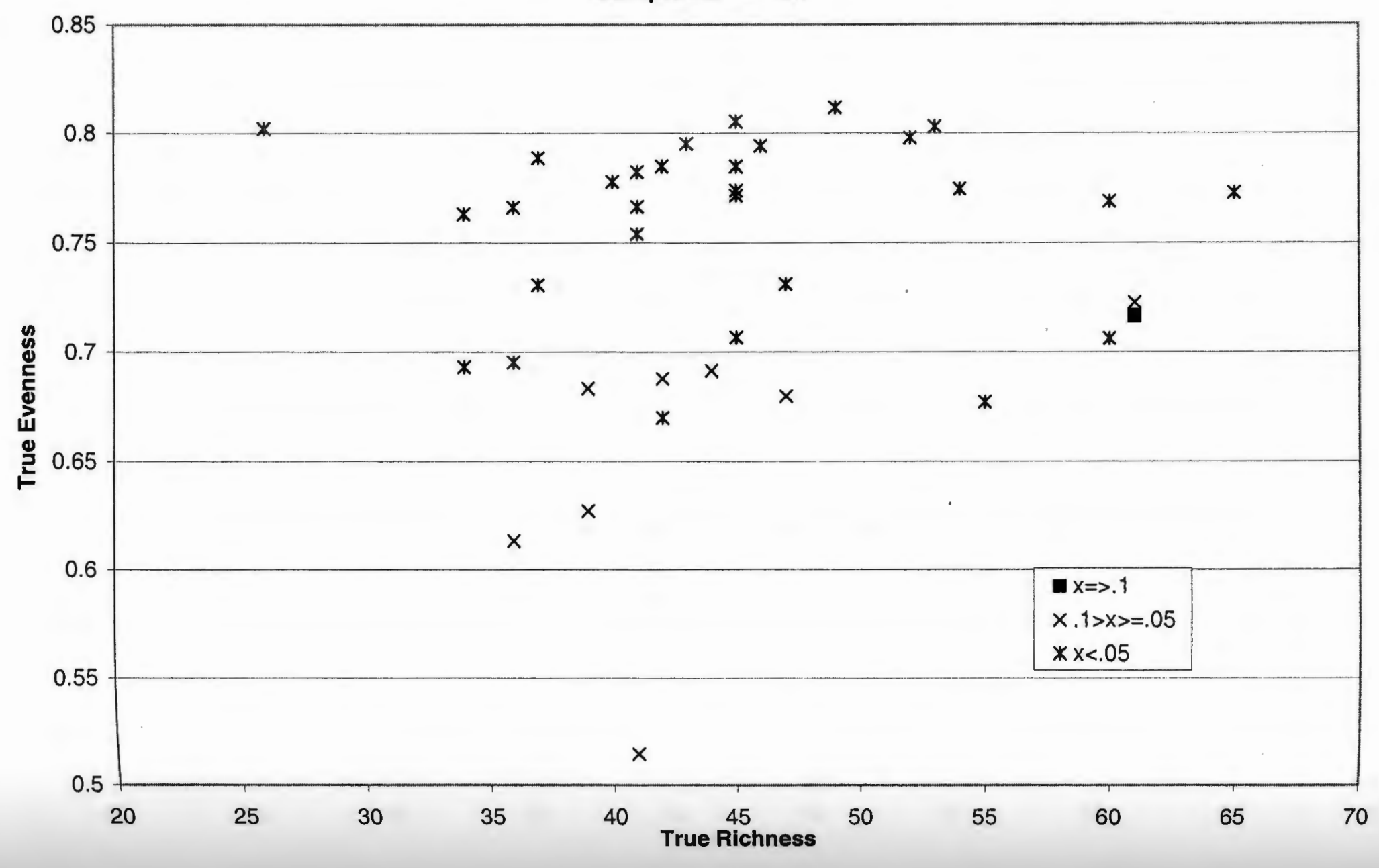


Figure 7 - Genus Level: True Richness vs. Alpha(Richness)

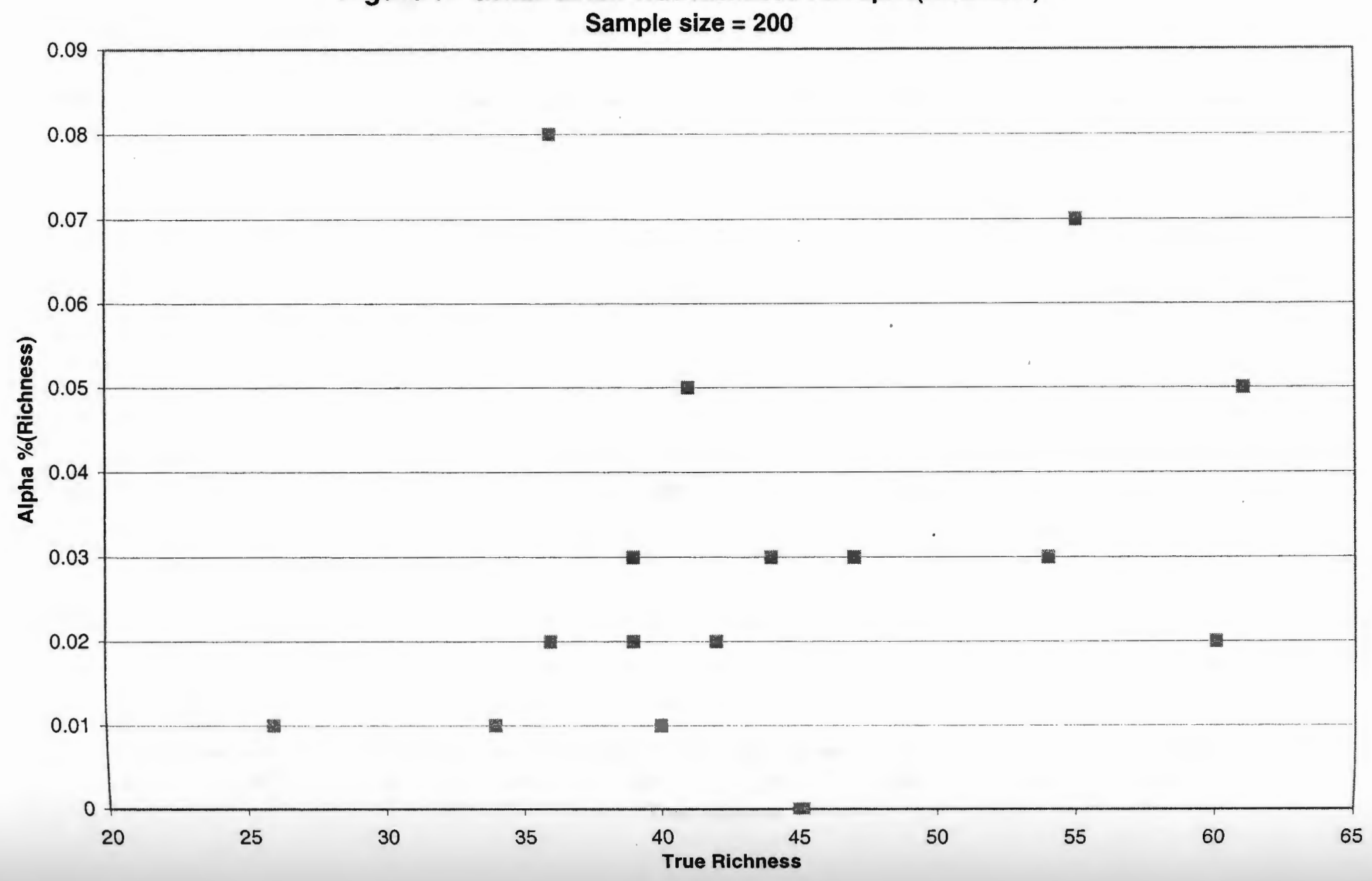


Figure 8 - Genus Level: True Richness vs. Alpha(CLI)

Sample size $=\mathbf{2 0 0}$

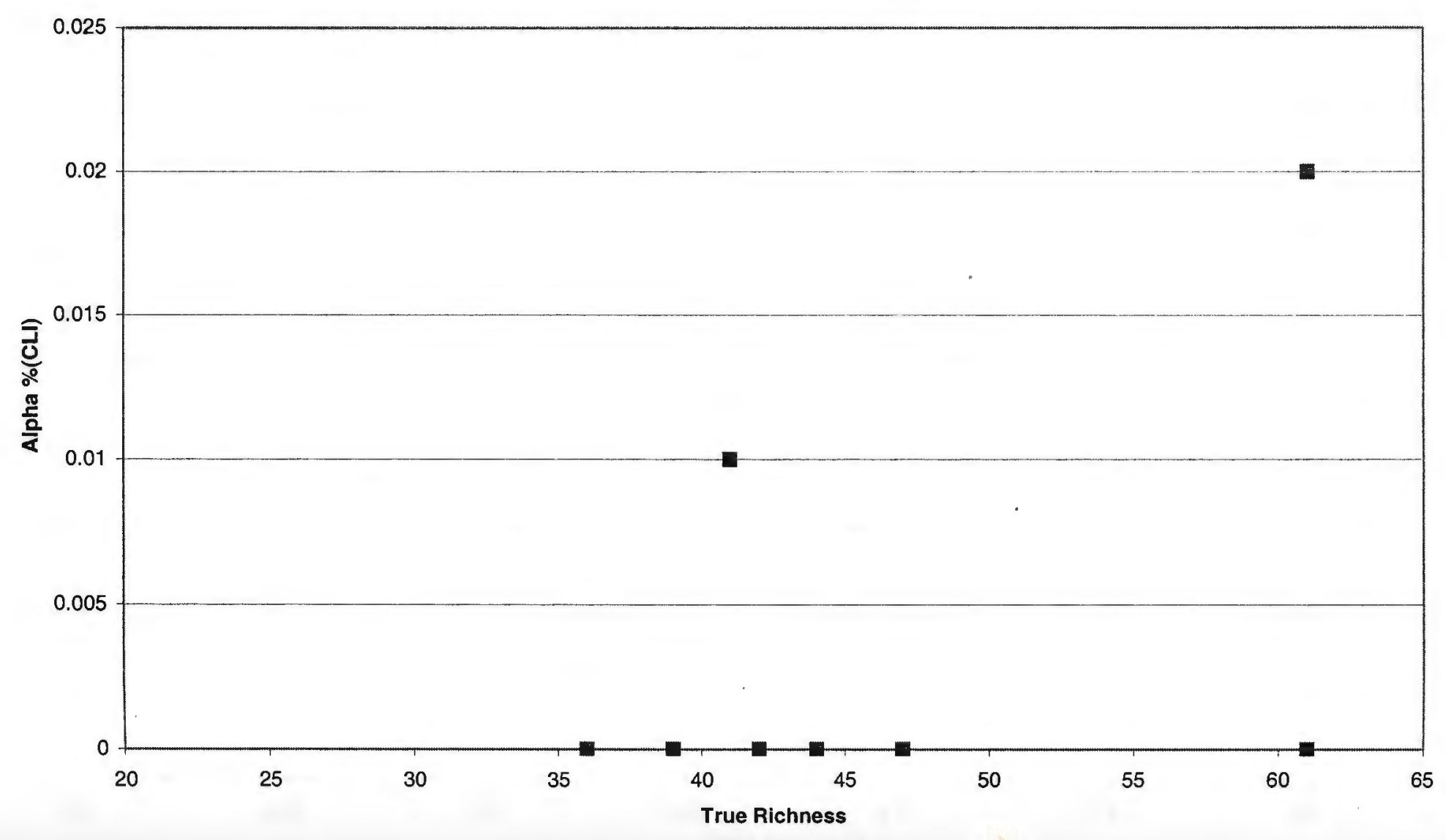




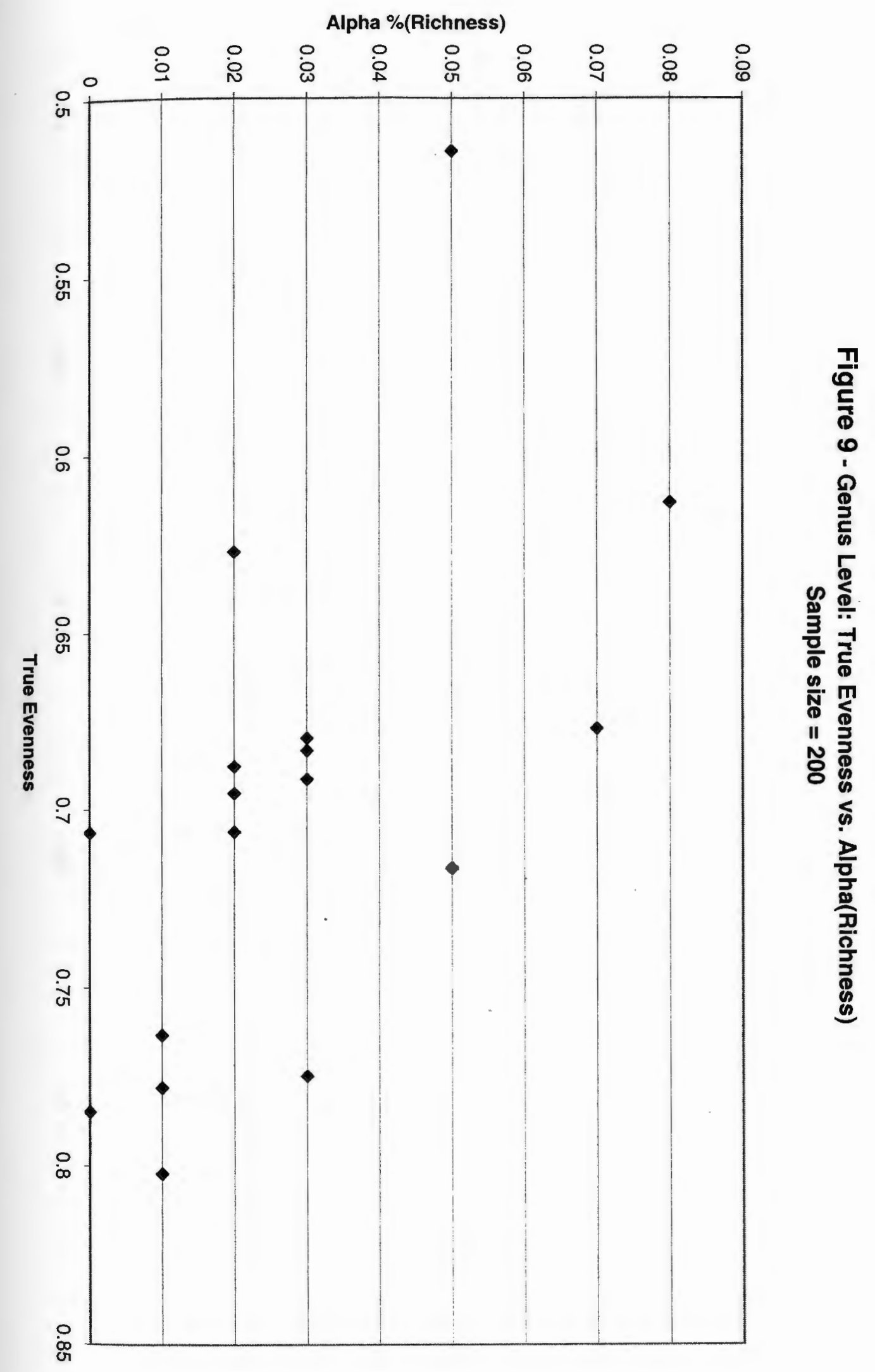




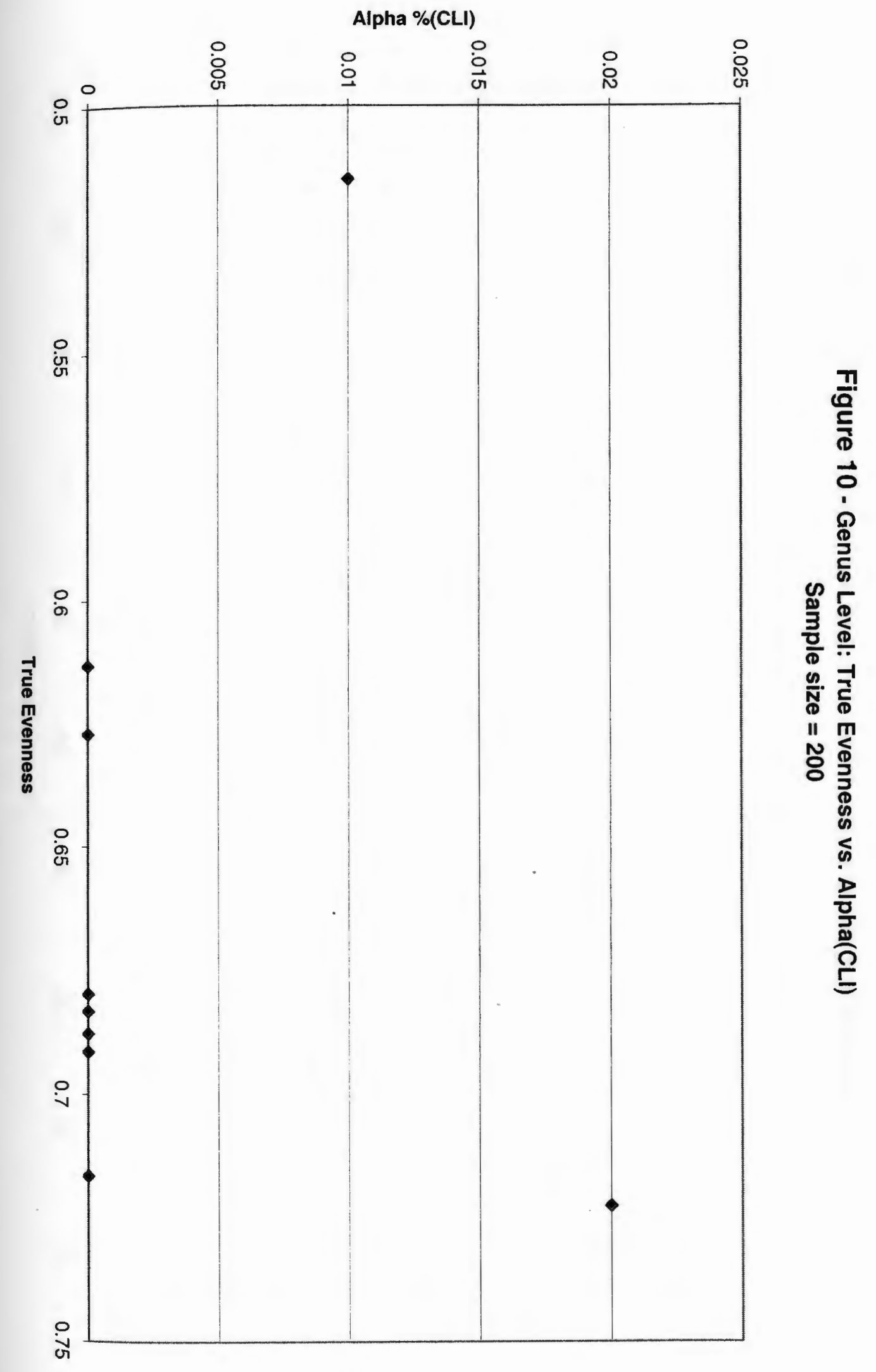


Figure 11 - Genus Level: True Richness vs. True Evenness(Alpha Richness)

Sample size $=200$

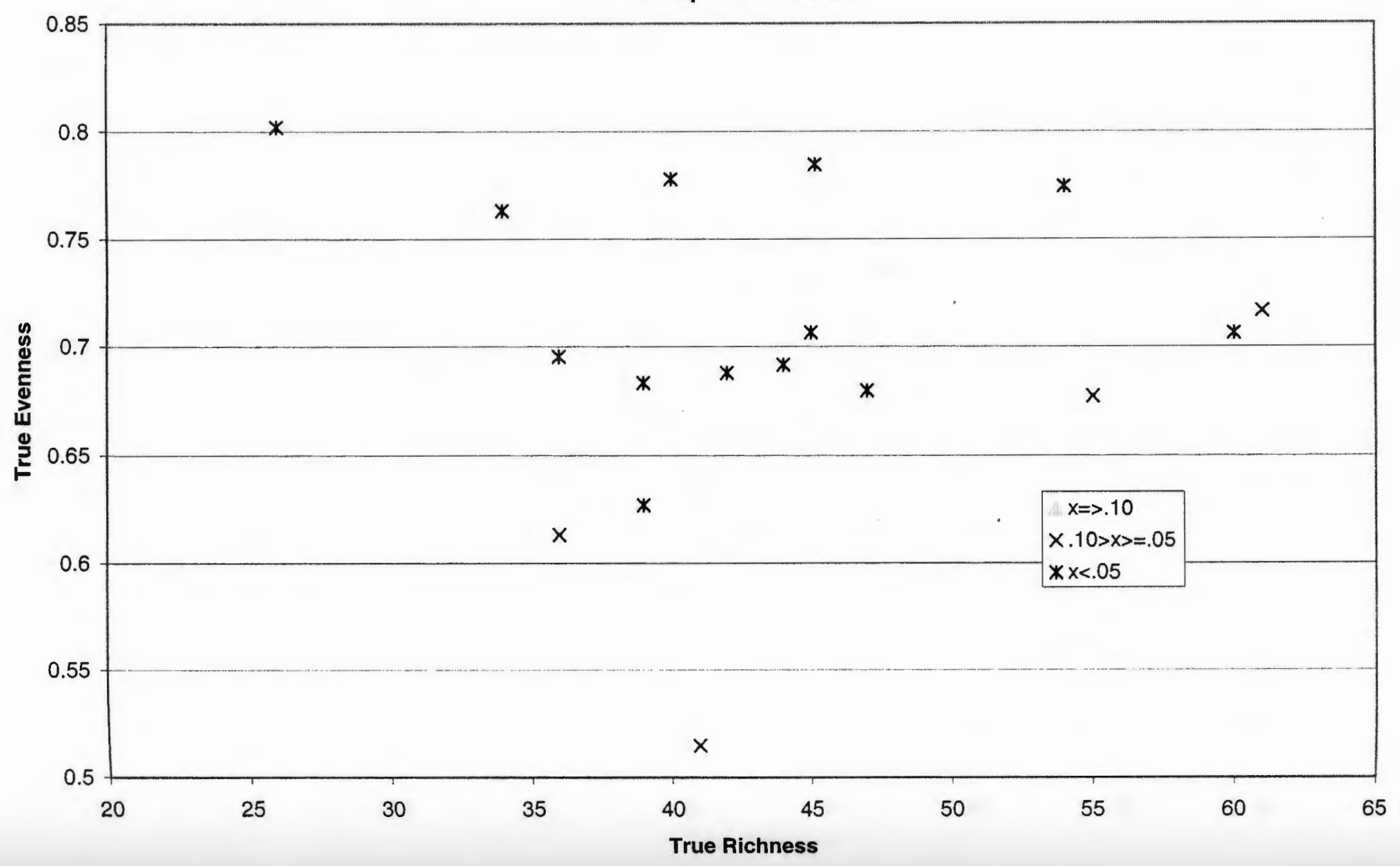


Figure 12 - Genus Level: True Richness vs. True Evenness(Alpha CLI) Sample size $=200$

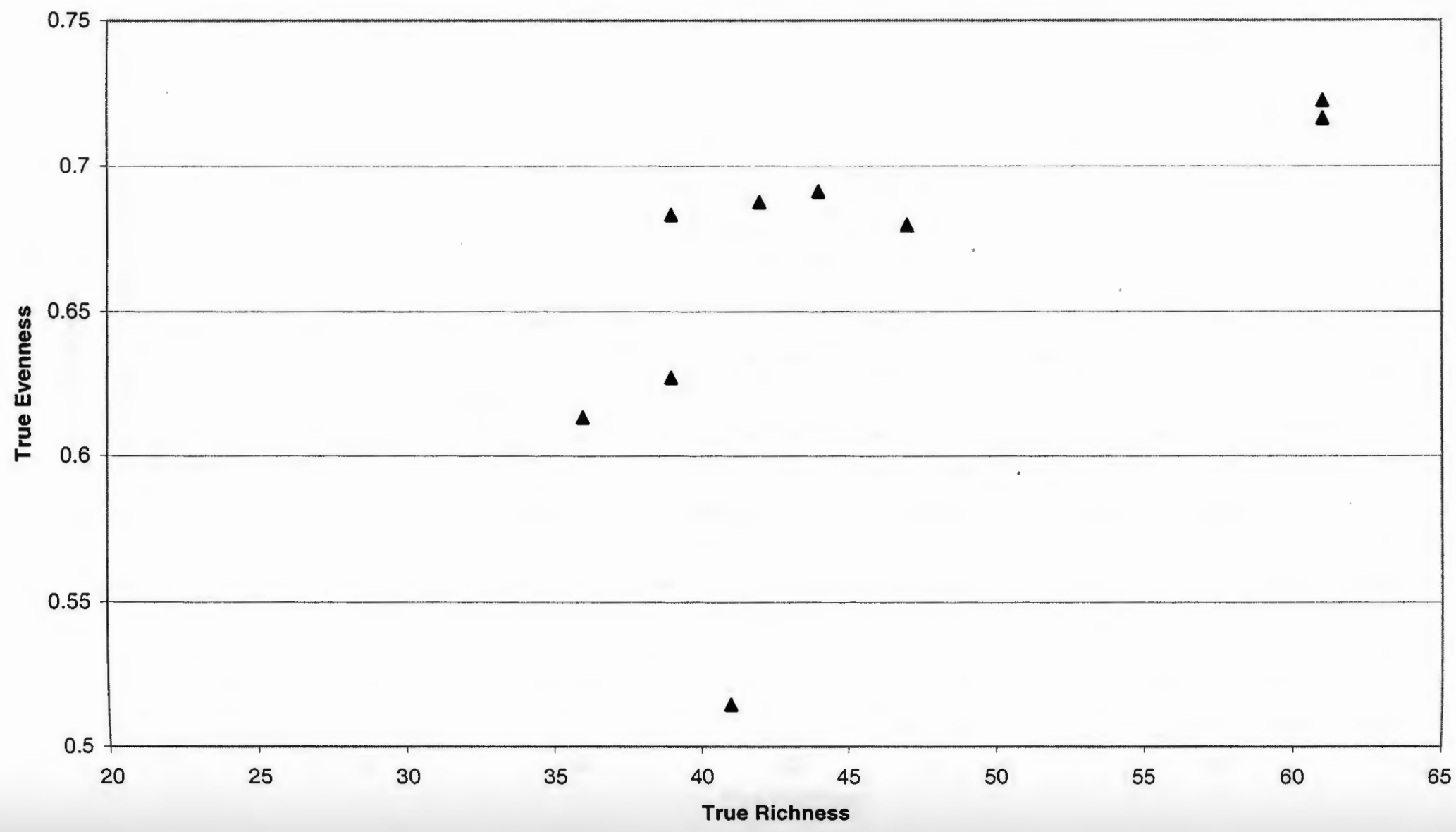


Figure 13 - Family Level: True Richness vs. Alpha(Richness)

Sample size $=100$

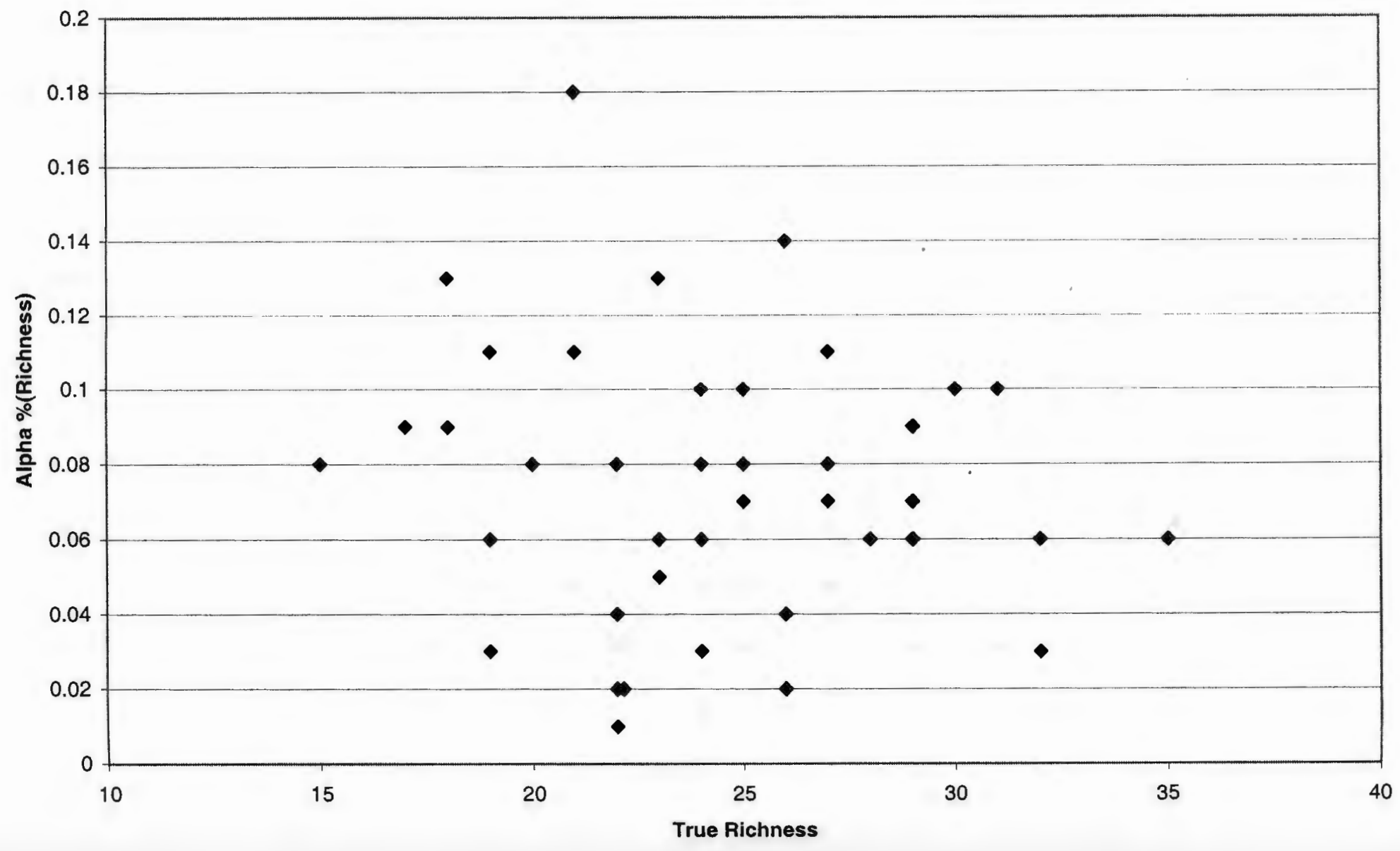


Figure 14 - Family Level: True Richness vs. Alpha(CLI)

Sample size $=100$

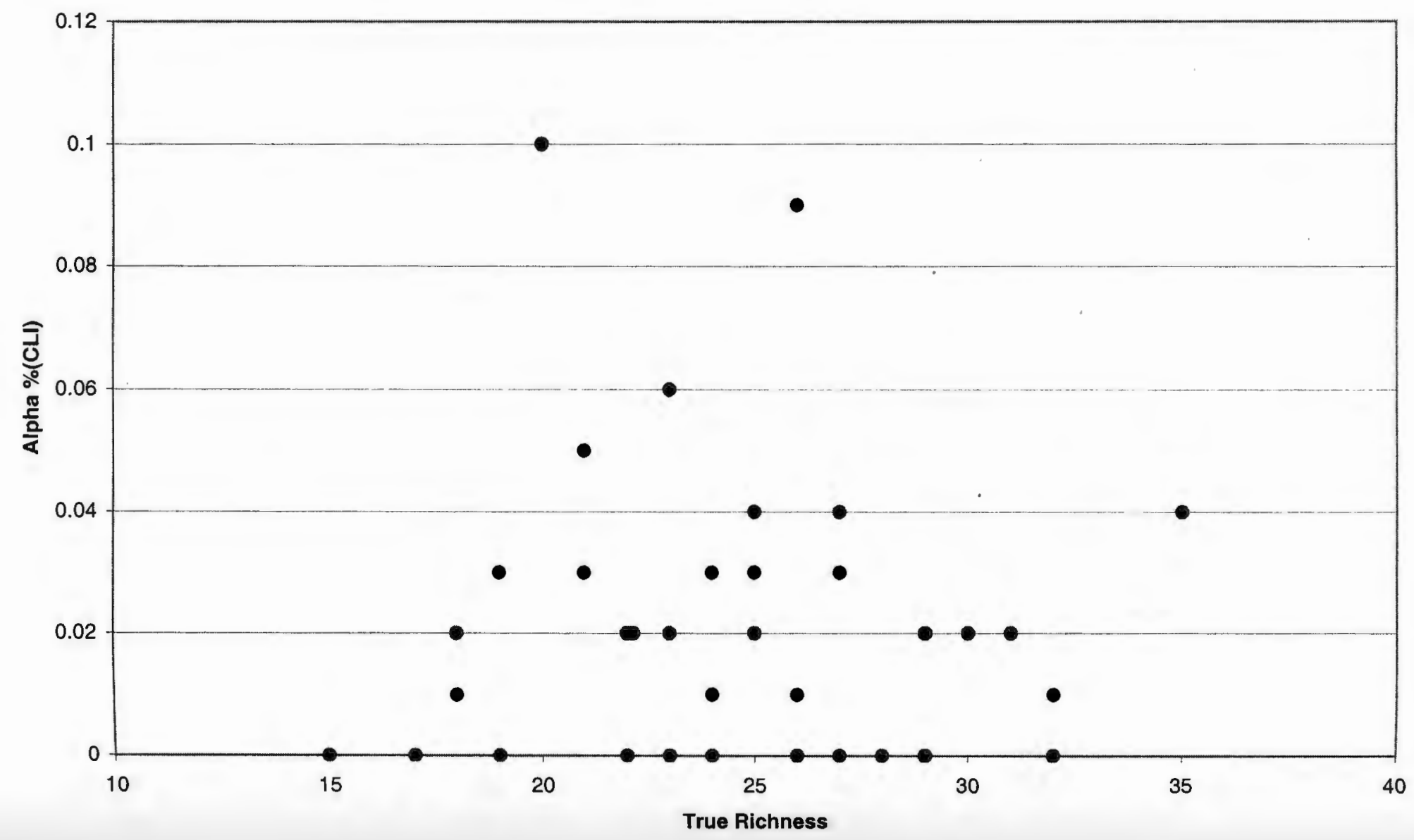


Figure 15 - Family Level: True Evenness vs. Alpha(Richness)

Sample size $=100$

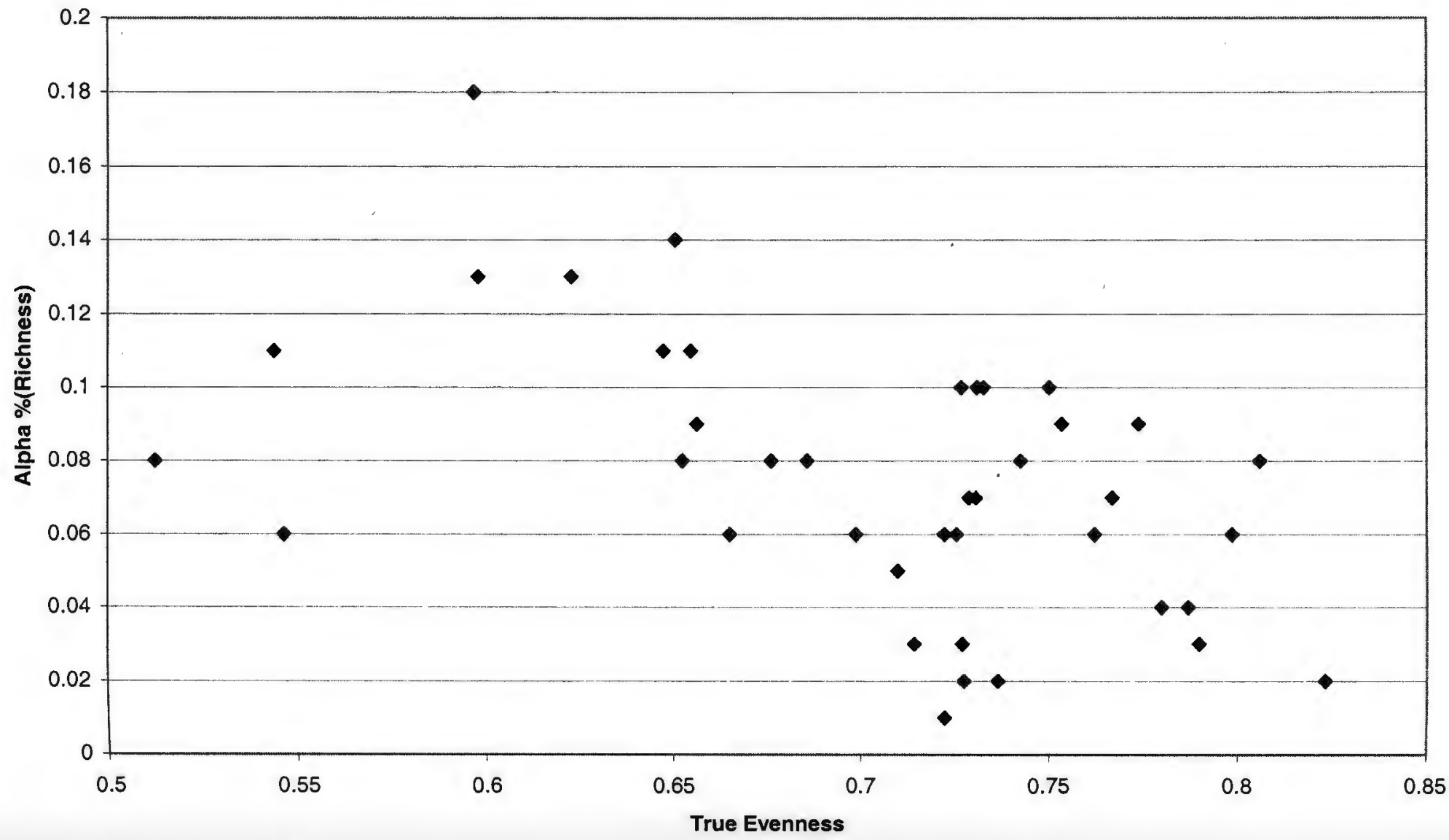


Figure 16 - Family Level: True Evenness vs. Alpha(CLI)

Sample size $=100$

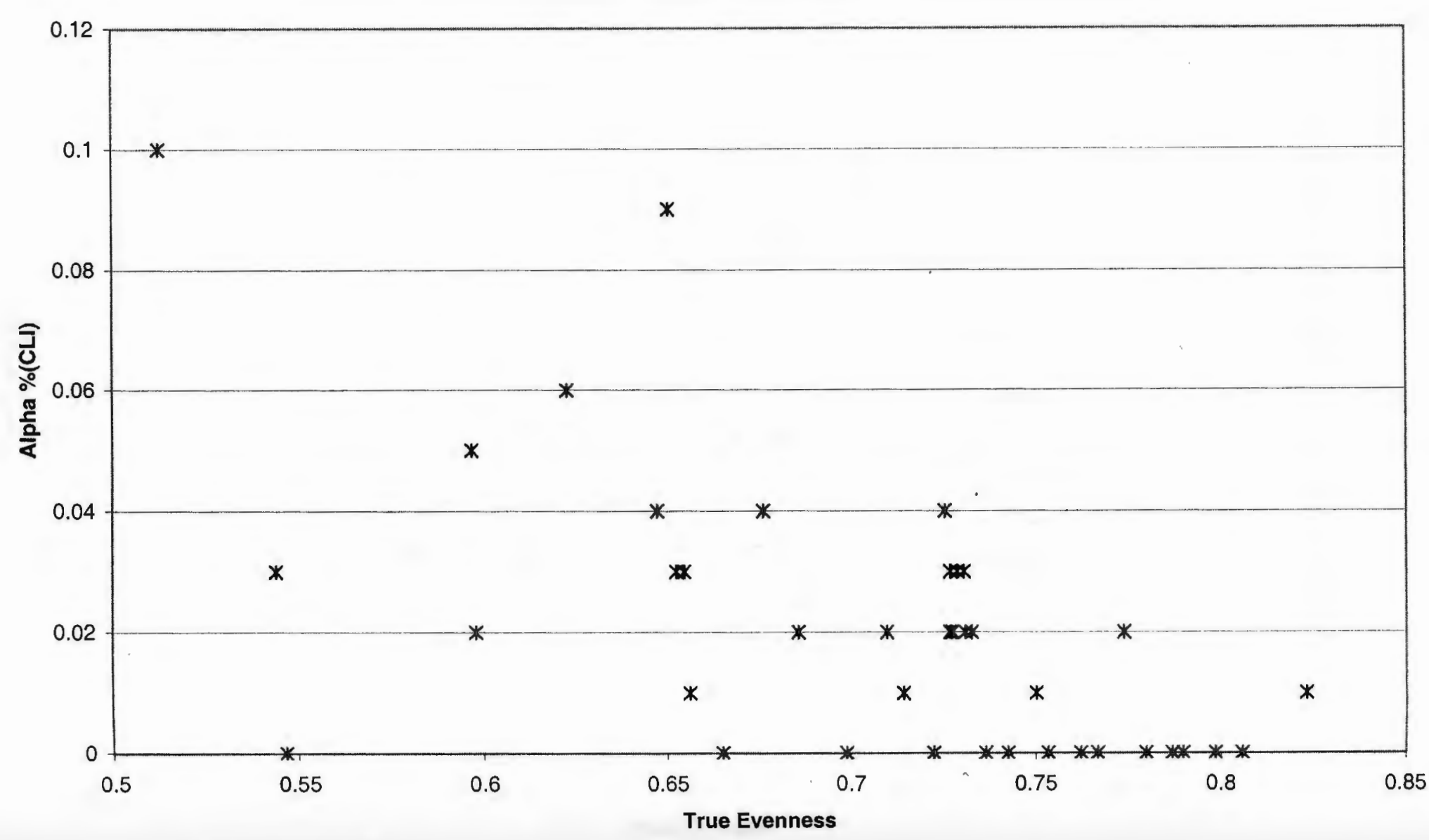


Figure 17 - Famlly Level: True Richness vs. True Evenness(Richness Alpha)

Sample size $=\mathbf{1 0 0}$

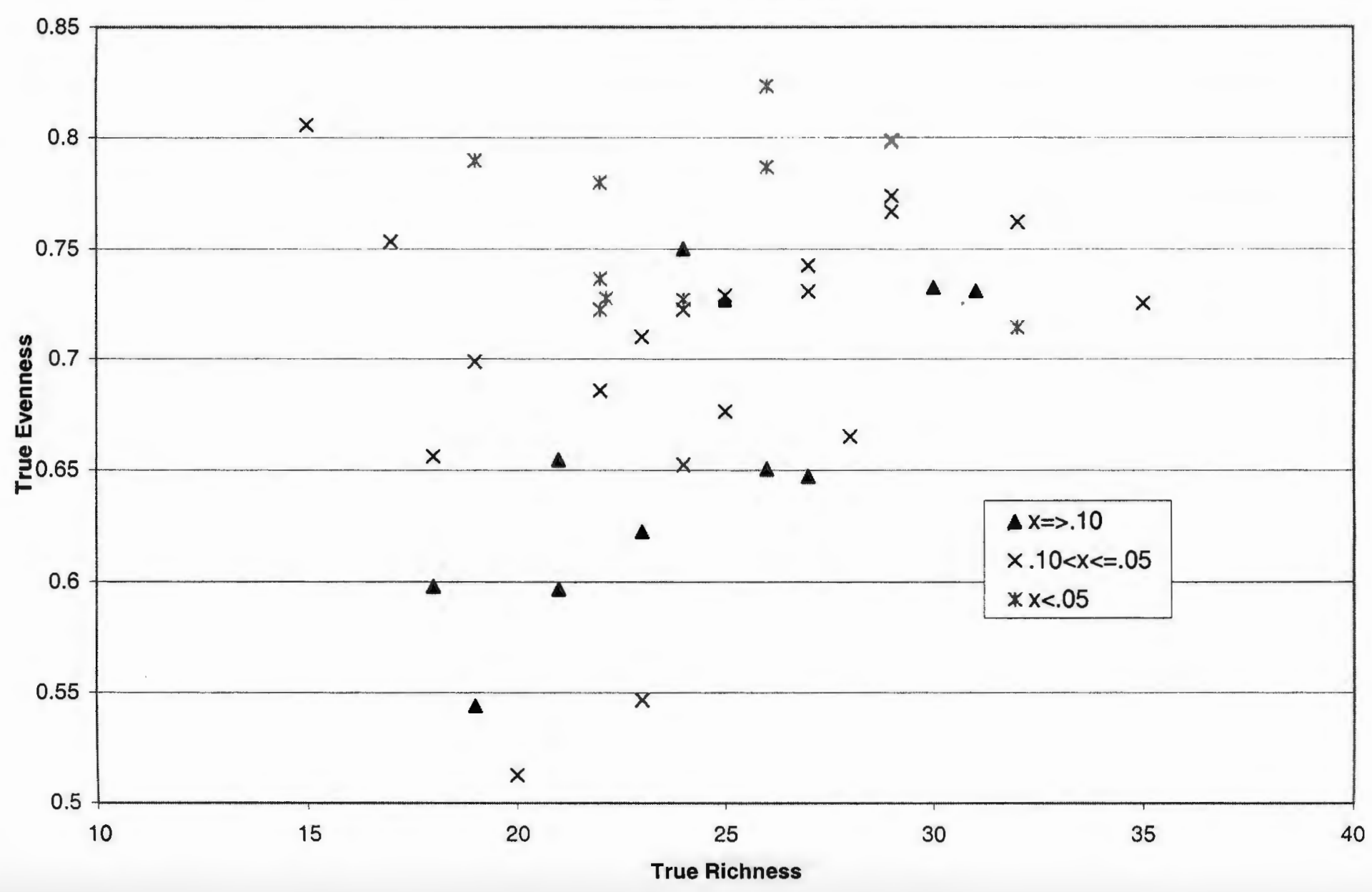


Figure 18 - Family Level: True Richness vs. True Evenness(CLI alpha)

$$
\text { Sample size }=100
$$

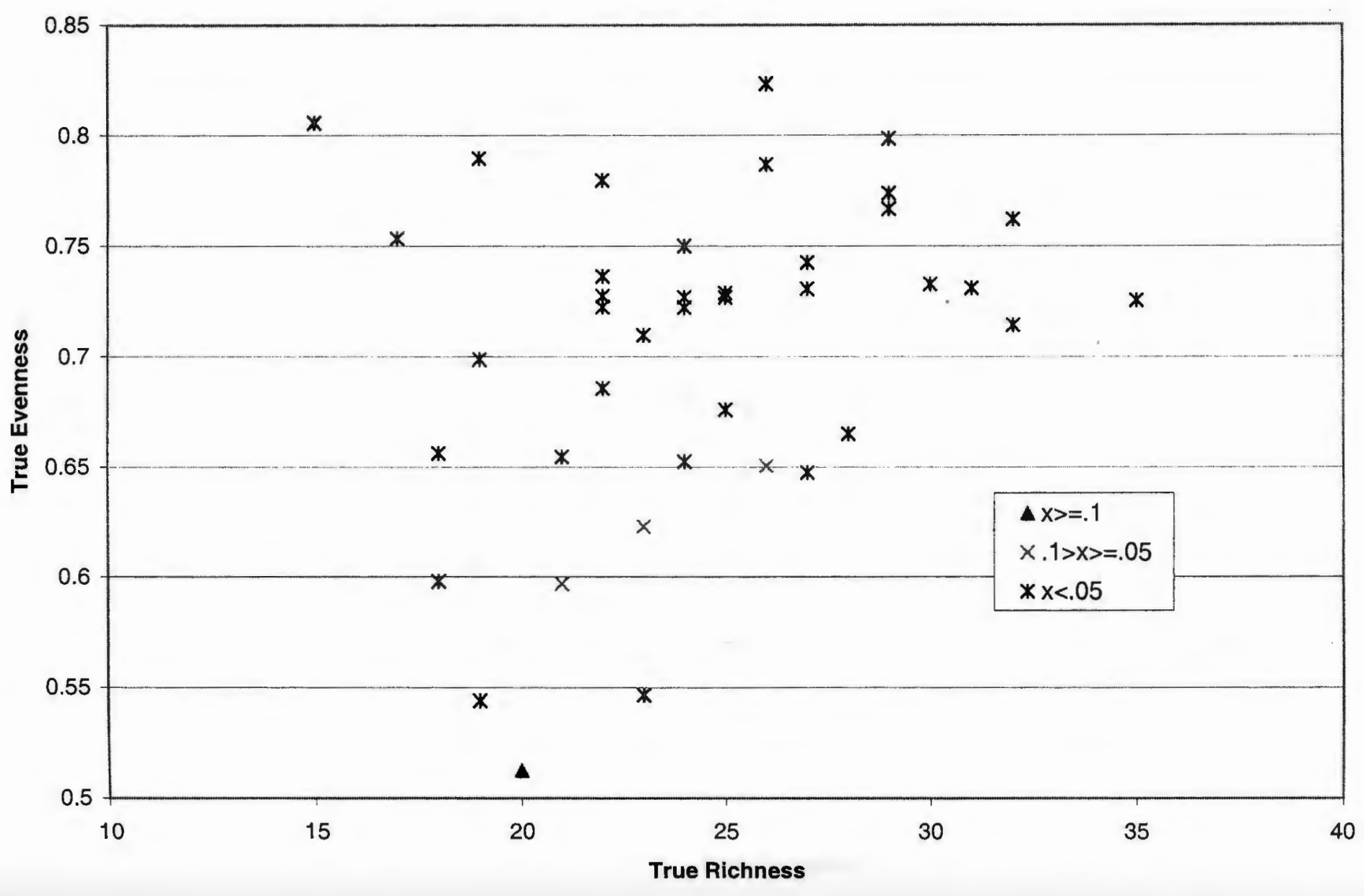


Figure 19 - Family Level: True Richness vs. Alpha(Richness)

Sample size $=\mathbf{2 0 0}$

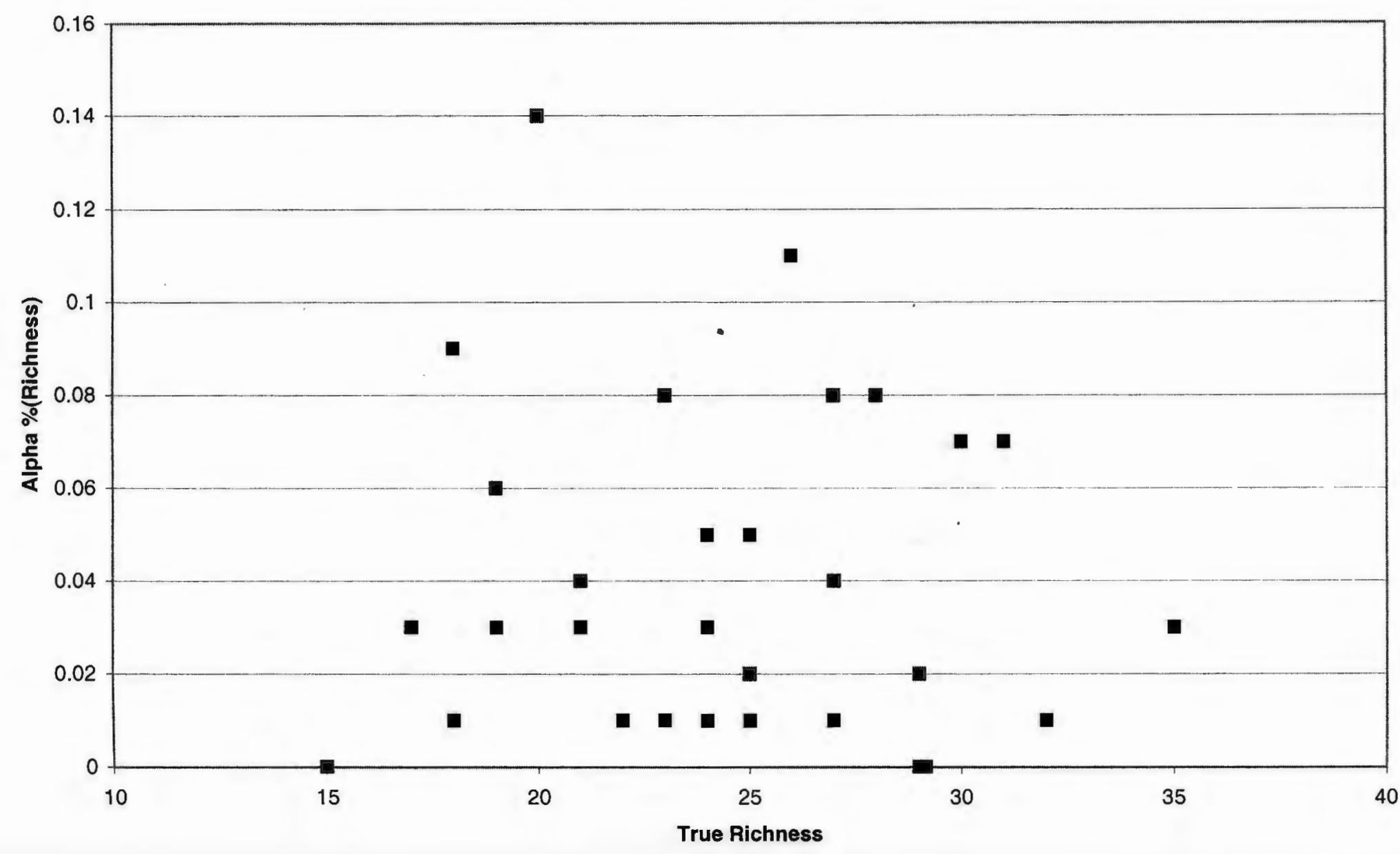


Figure 20 - Family Level: True Evenness vs. Alpha(Richness)

Sample size $=200$

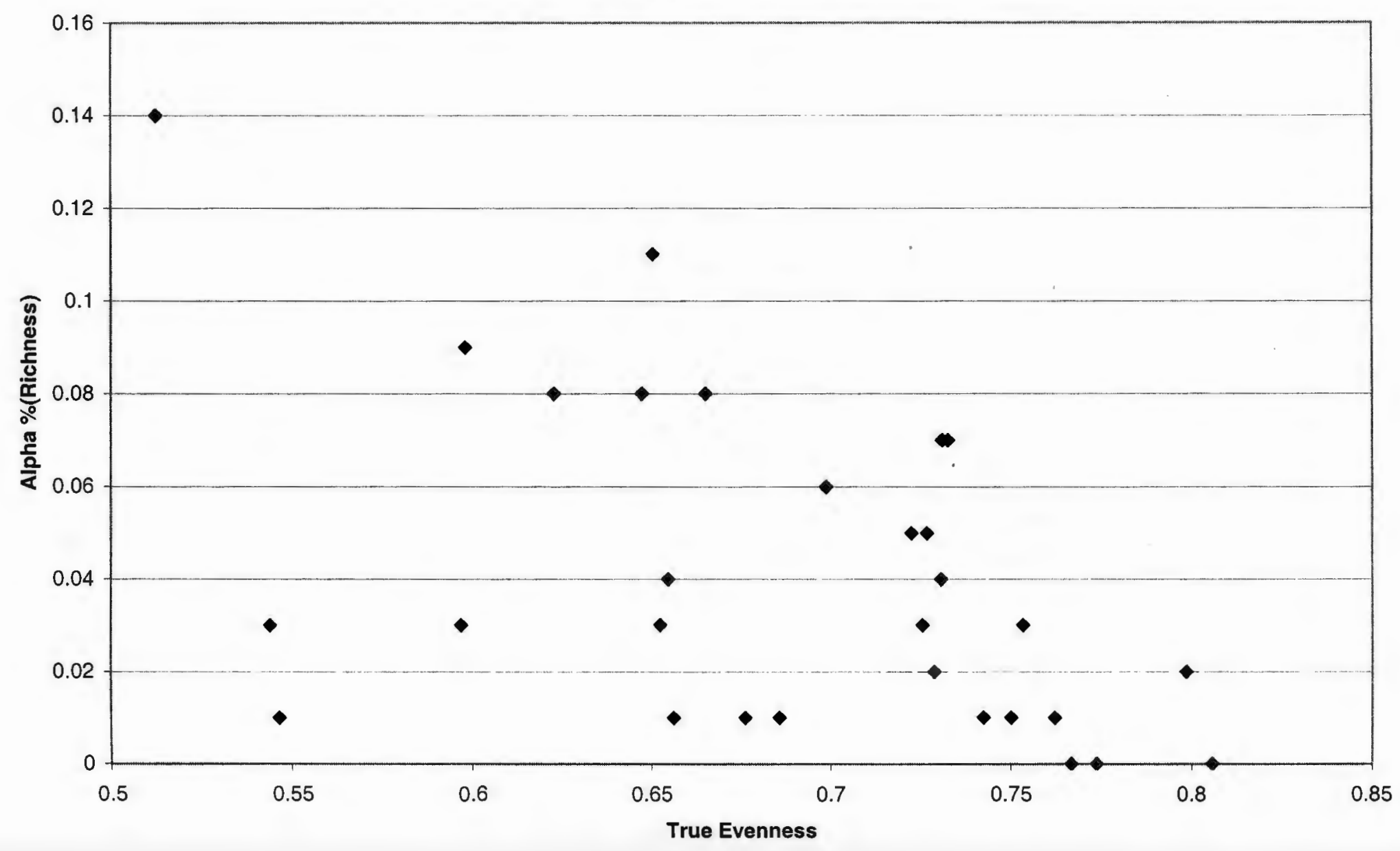


Figure 21 - Family Level: True Richness vs. True Evenness(Richness Alpha)

Sample size $=\mathbf{2 0 0}$




Figure 22 - Family Level: True Richness vs. Alpha(Richness)

Sample size $=\mathbf{3 0 0}$






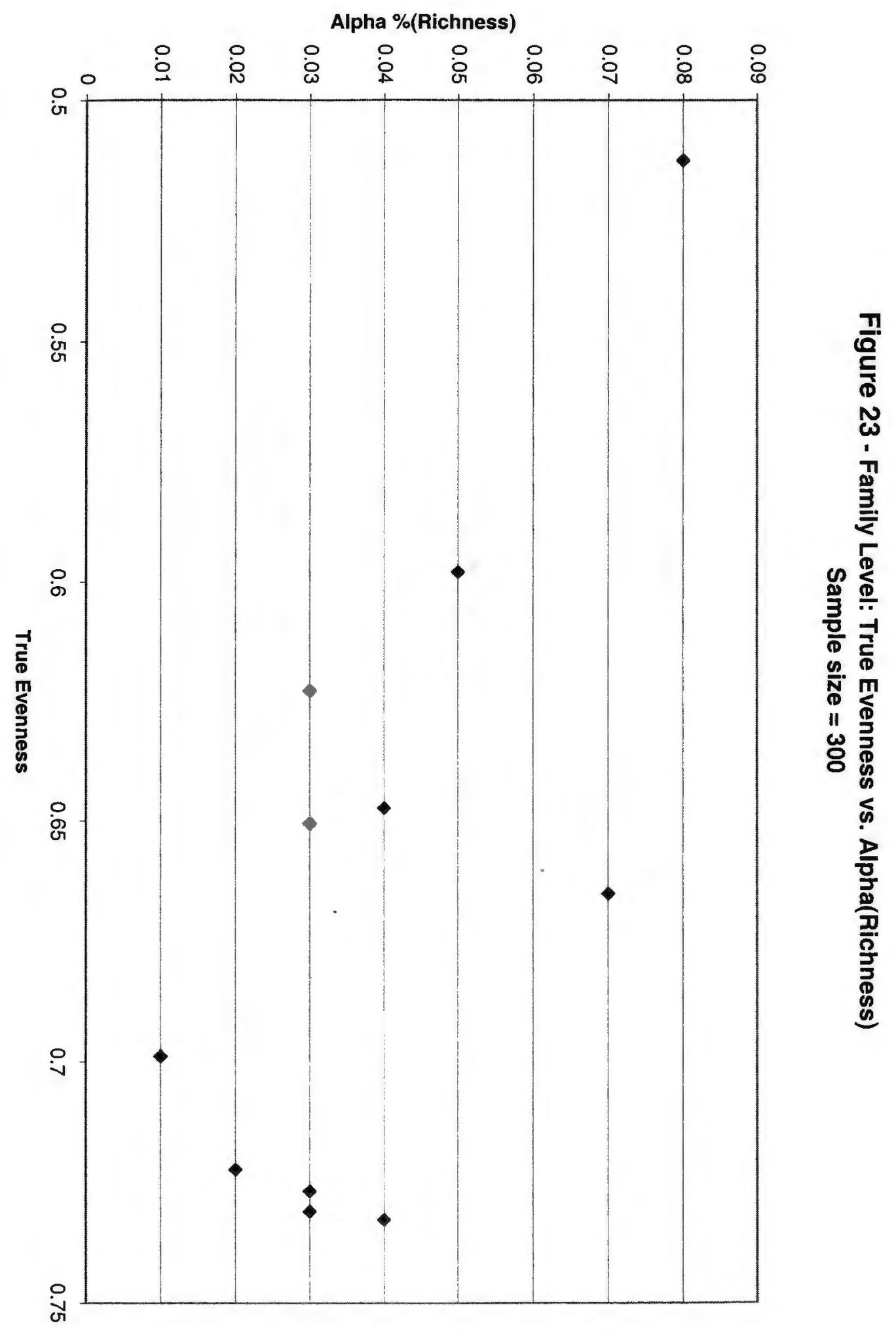


Figure 24 - Genus level: True Richness vs. Alpha(Richness) $=0.75$

Sample size $=100$




Figure 25 - Genus Level: True Evenness vs. Alpha(Richness) $=0.75$

Sample size $=100$

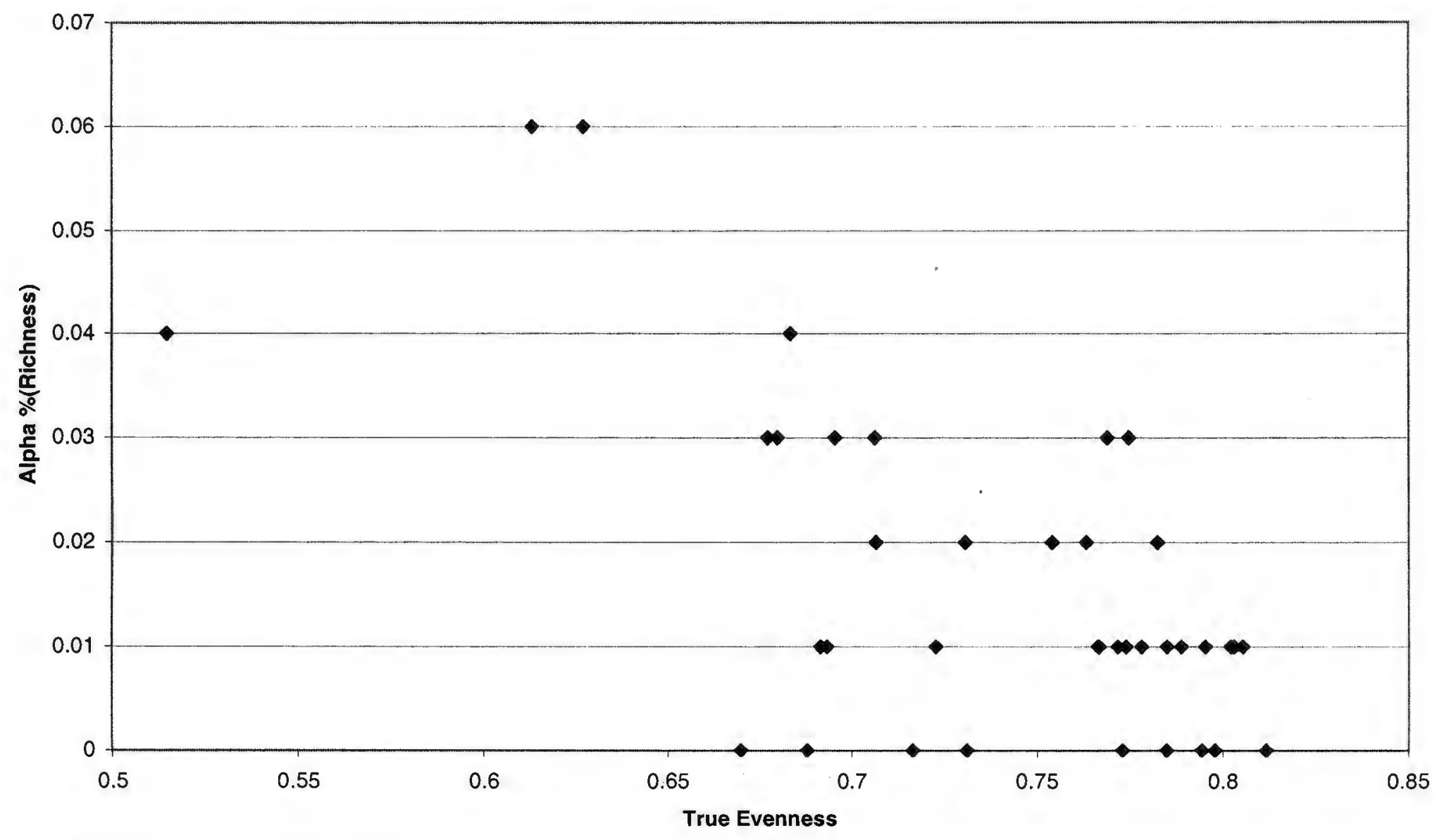


Figure 26 - Genus Level: True Richness vs. Alpha(CLI) $=0.55$

Sample size $=100$

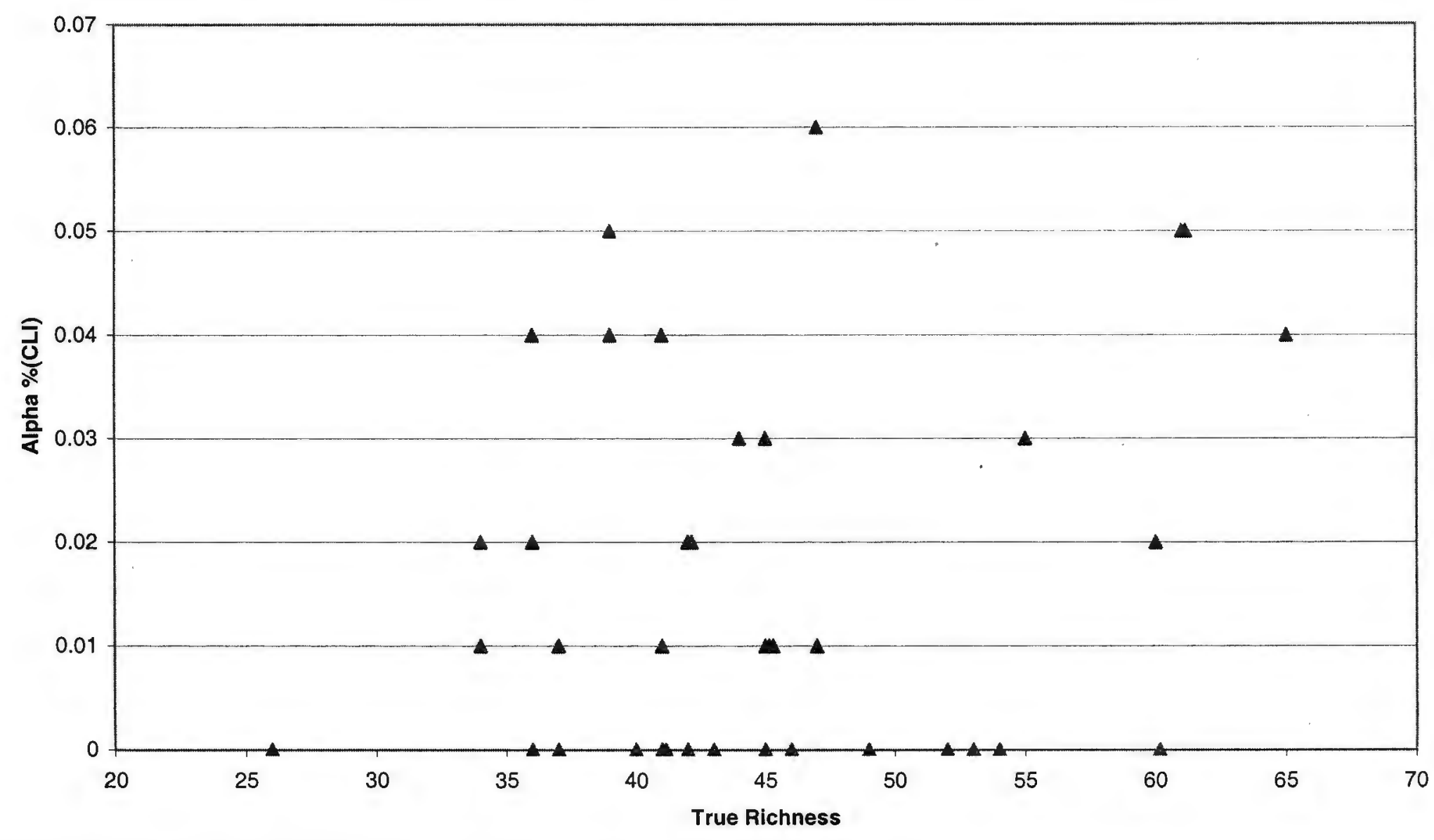




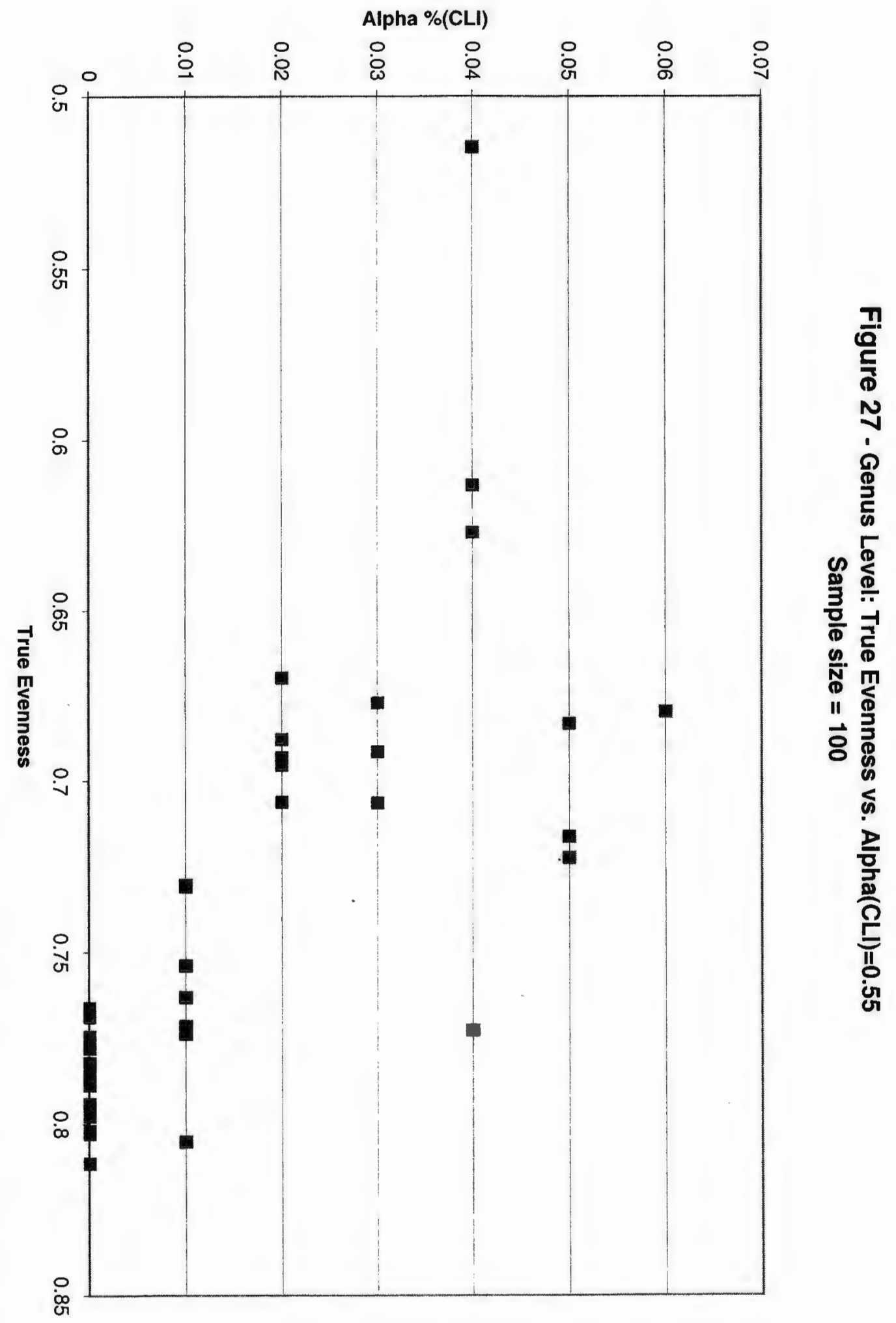


Figure 28 - Genus Level: True Richness vs. Alpha(CLI) $=0.60$

Sample size $=100$

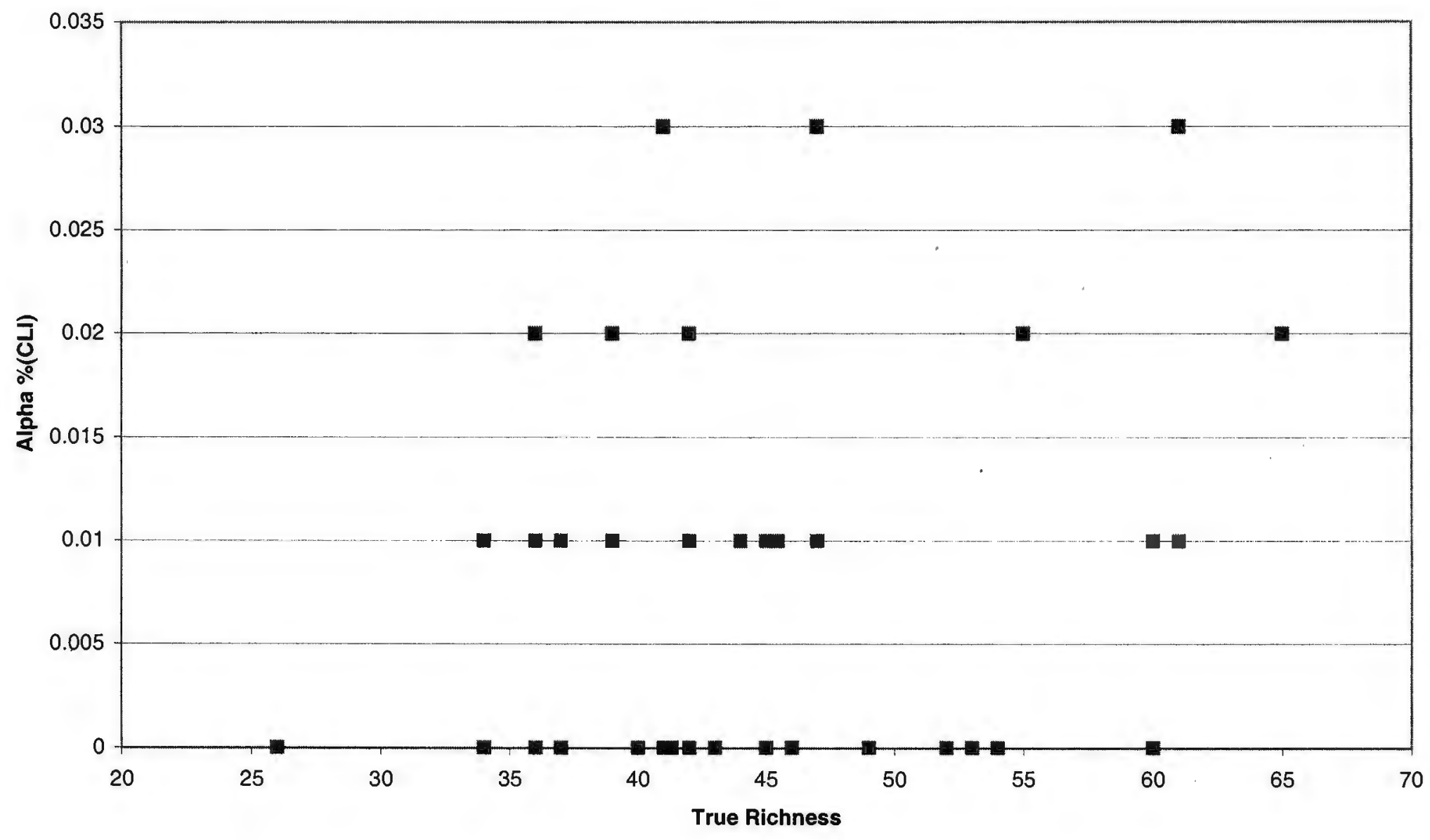




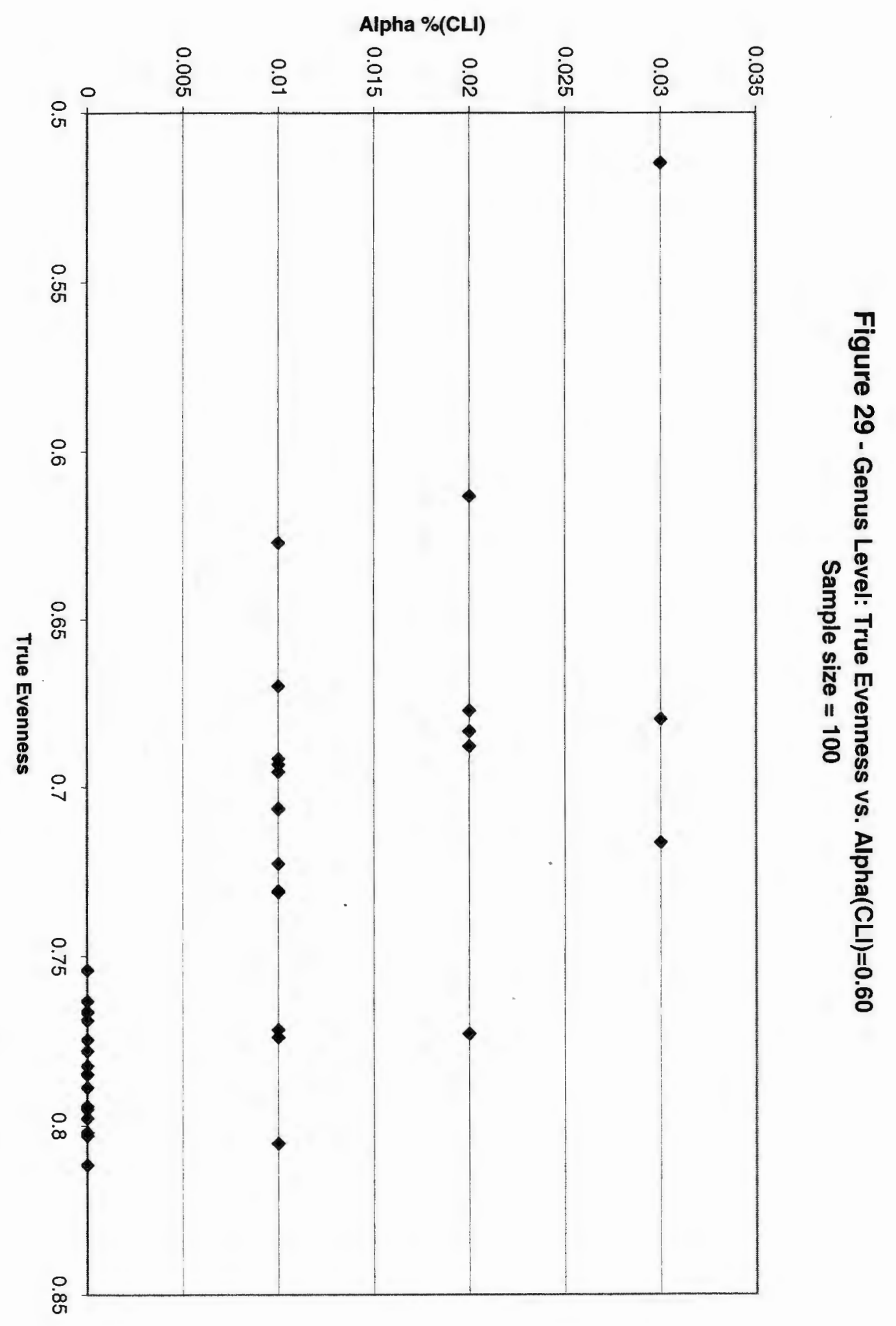


Figure 30 - Family Level: True Richness vs. Alpha(Richness) $=0.75$

Sample size $=100$

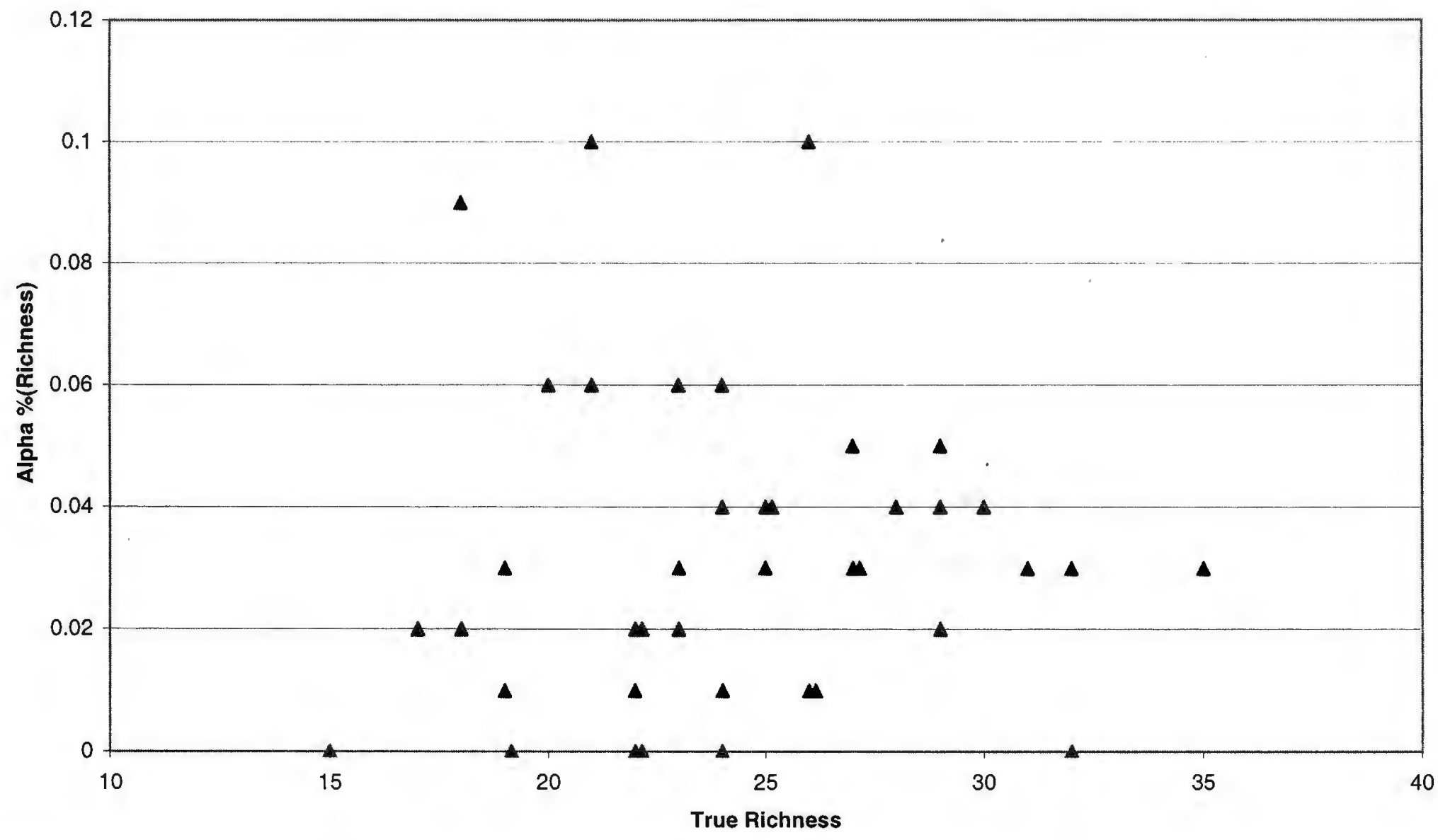


Figure 31 - Family Level: True Evenness vs. Alpha(Richness) $=0.75$

Sample size $=100$

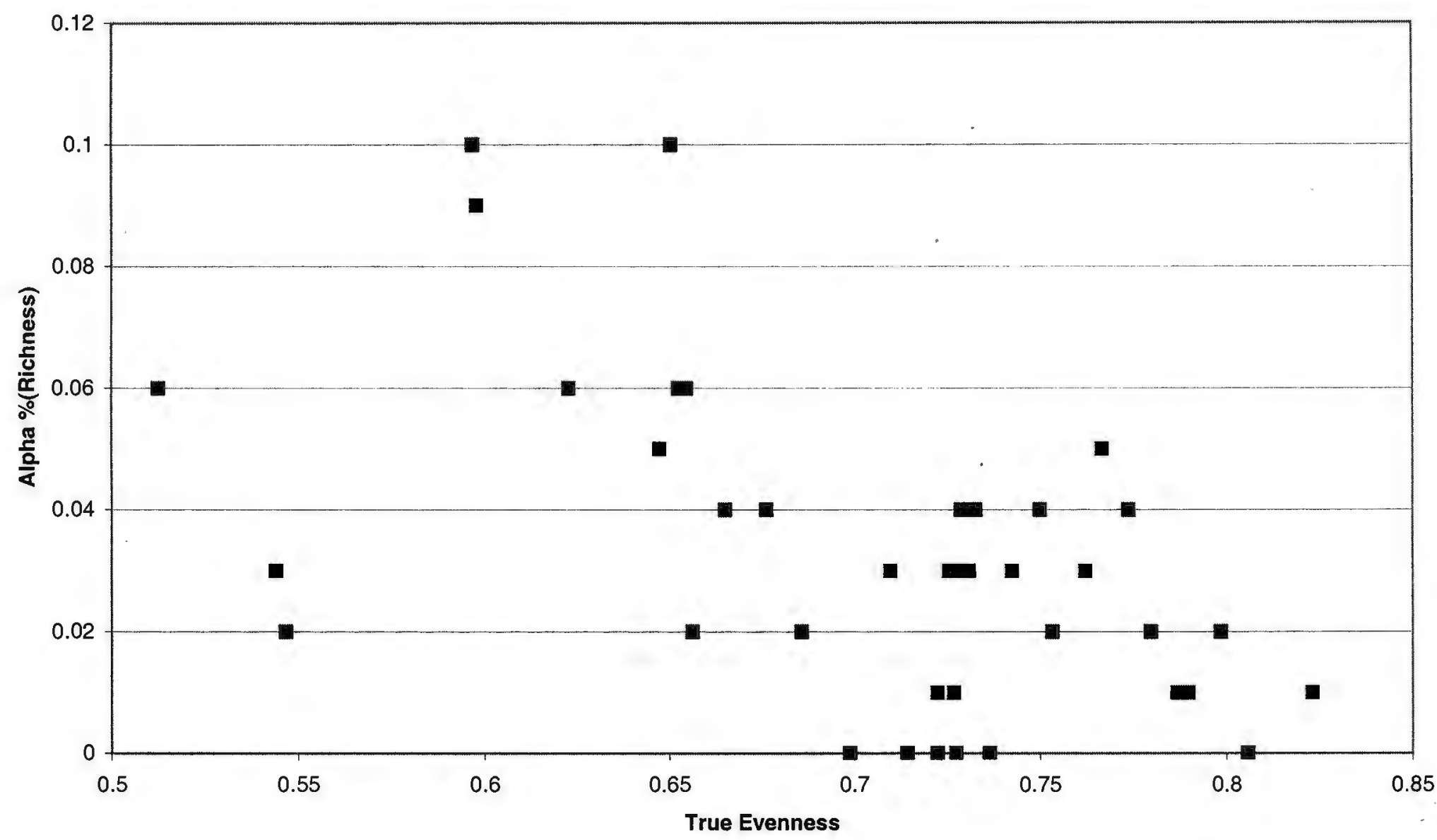


Figure 32 - Family Level: True Richness vs. Alpha(Richness) $=0.70$

Sample size $=100$

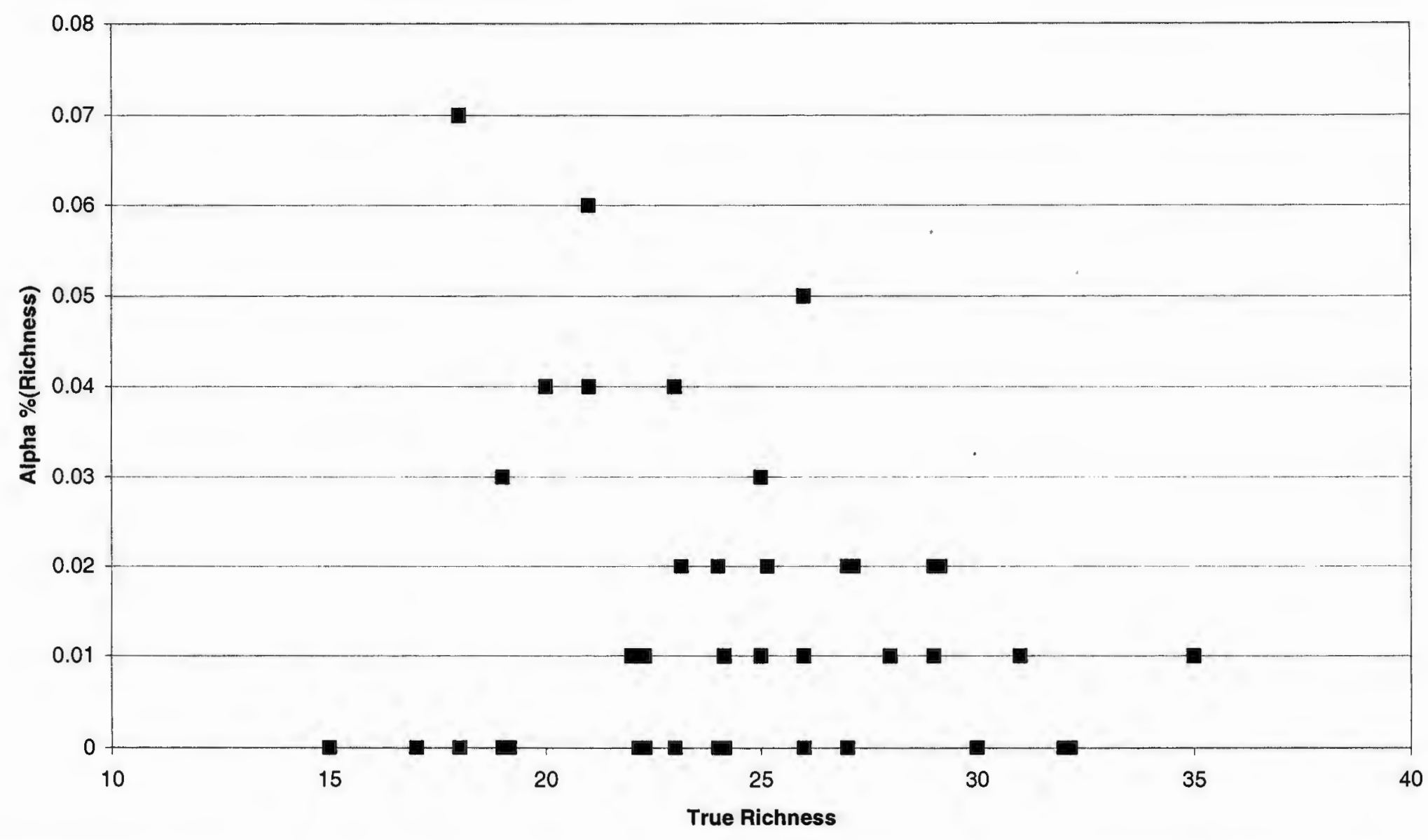




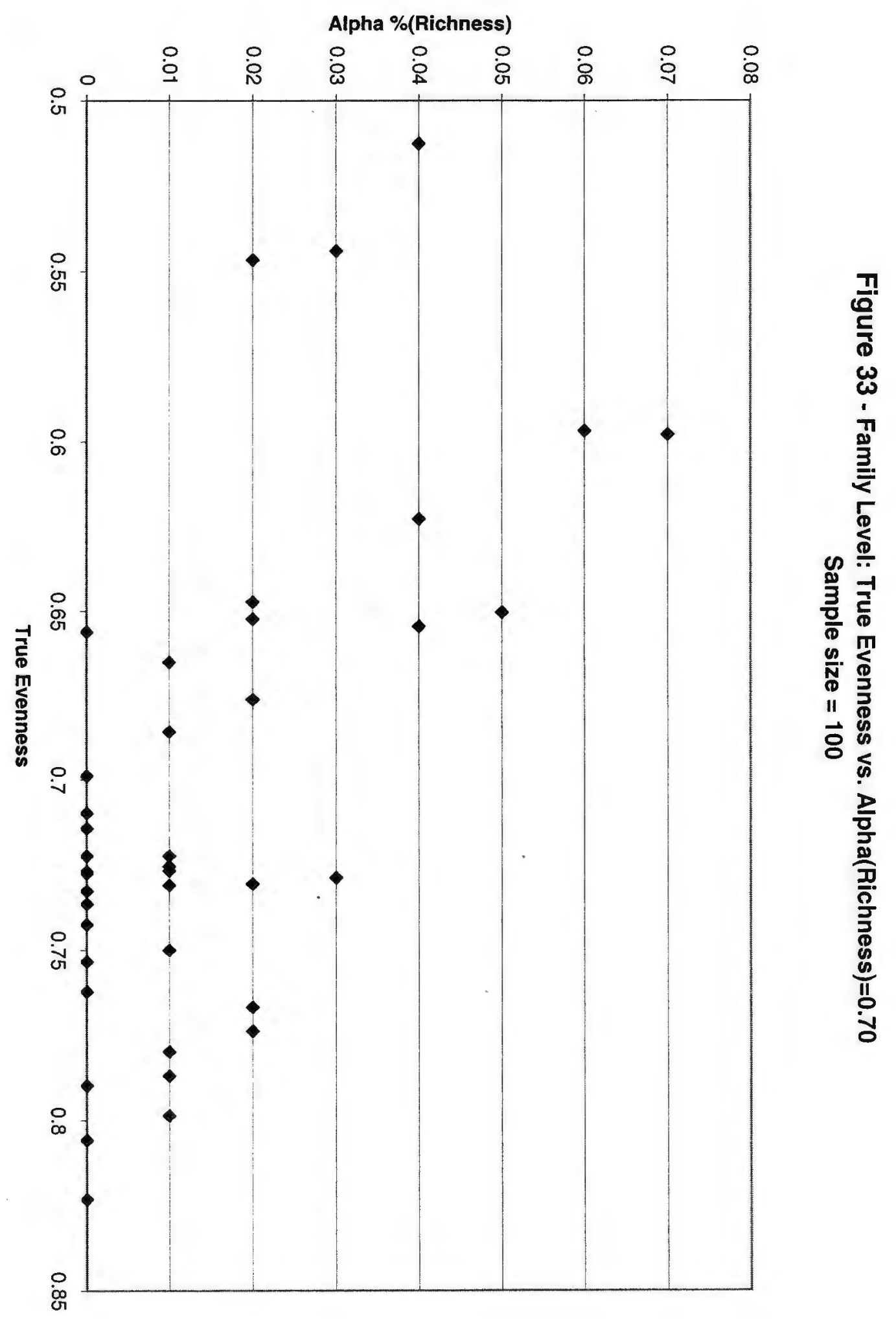


Figure 34 - Family Level: True Richness vs. Alpha(CLI) $=0.55$

Sample size $=100$

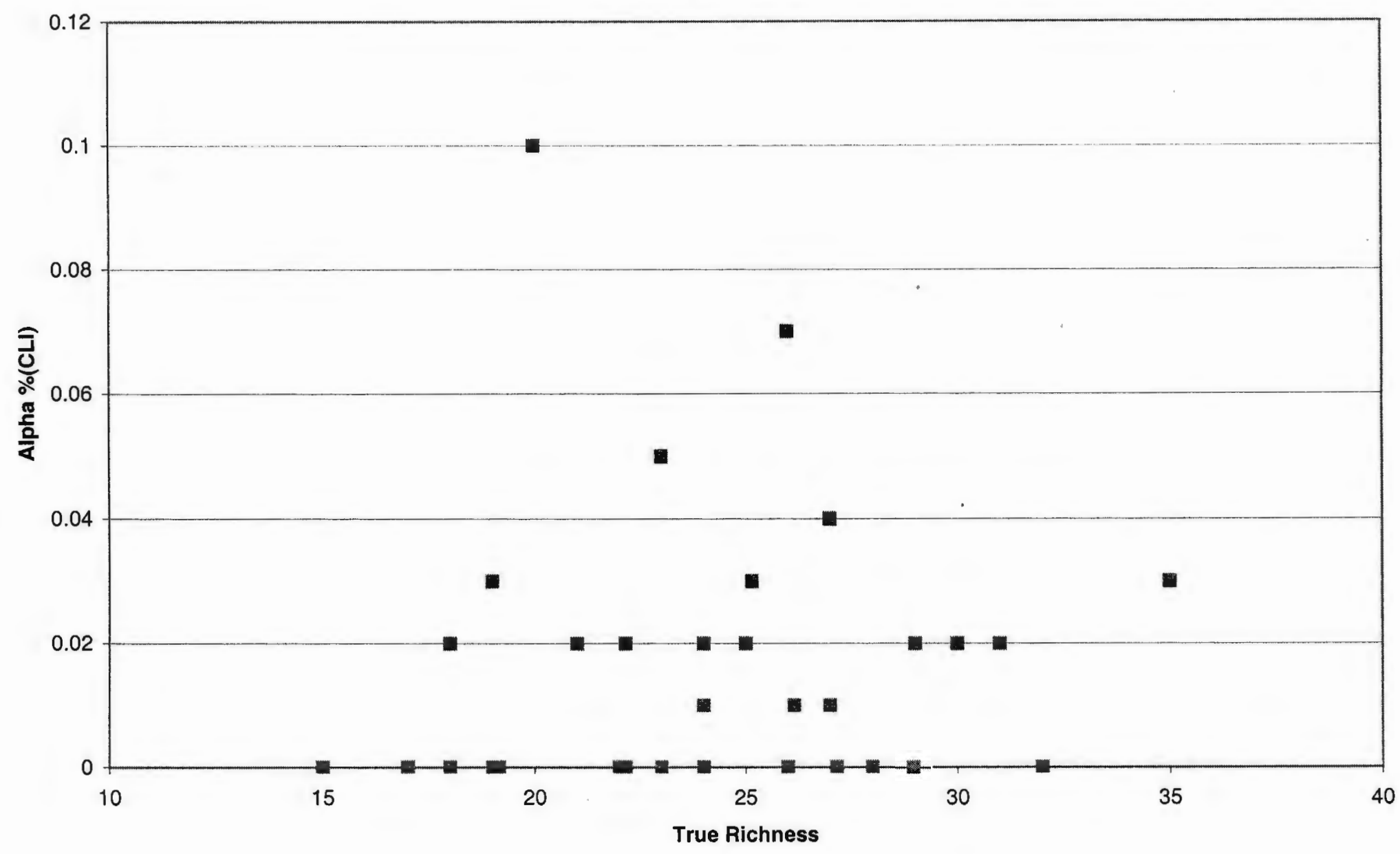




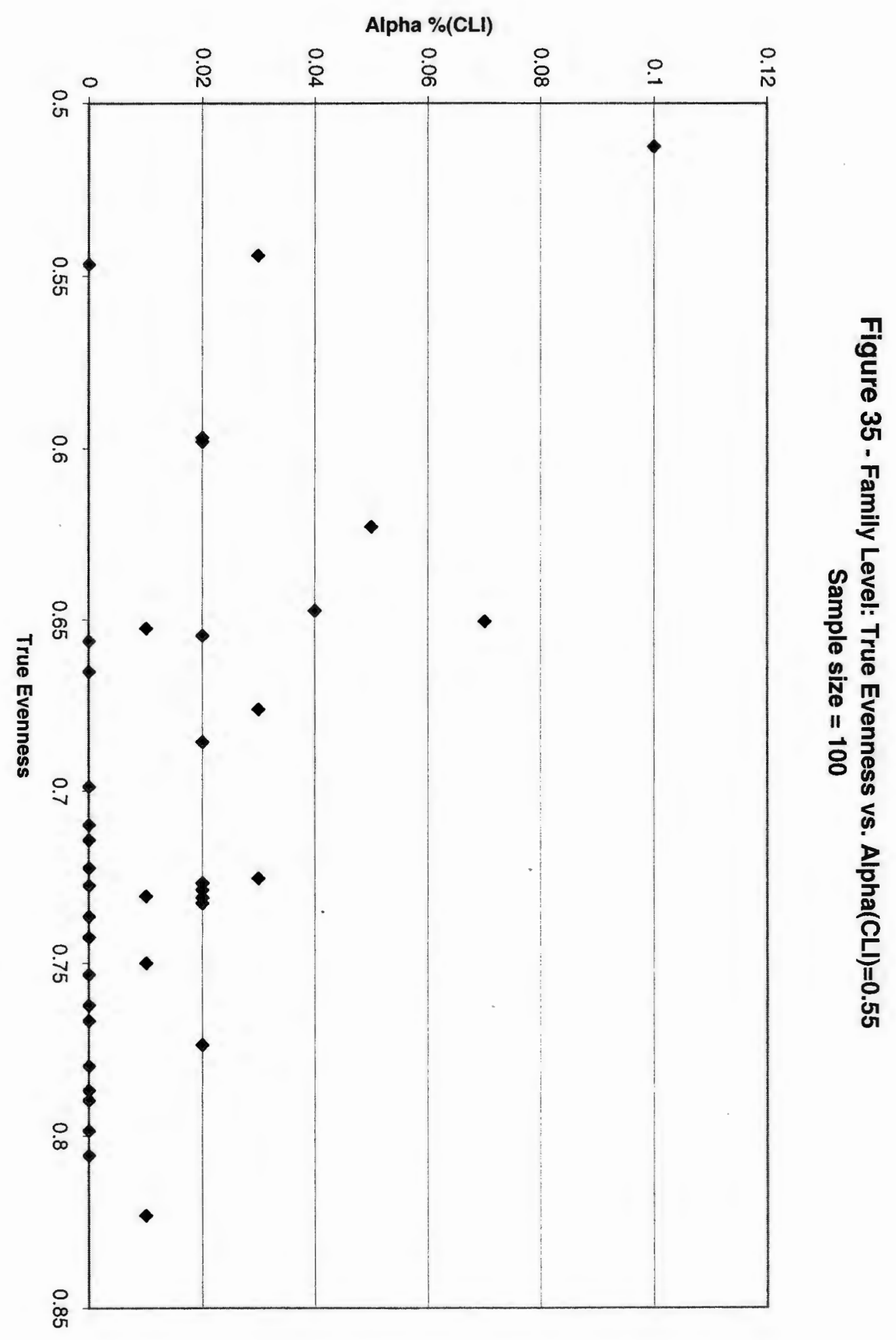


Figure 36 - Family Level: True Richness vs. Alpha(CLI) $=0.60$

Sample size $=100$

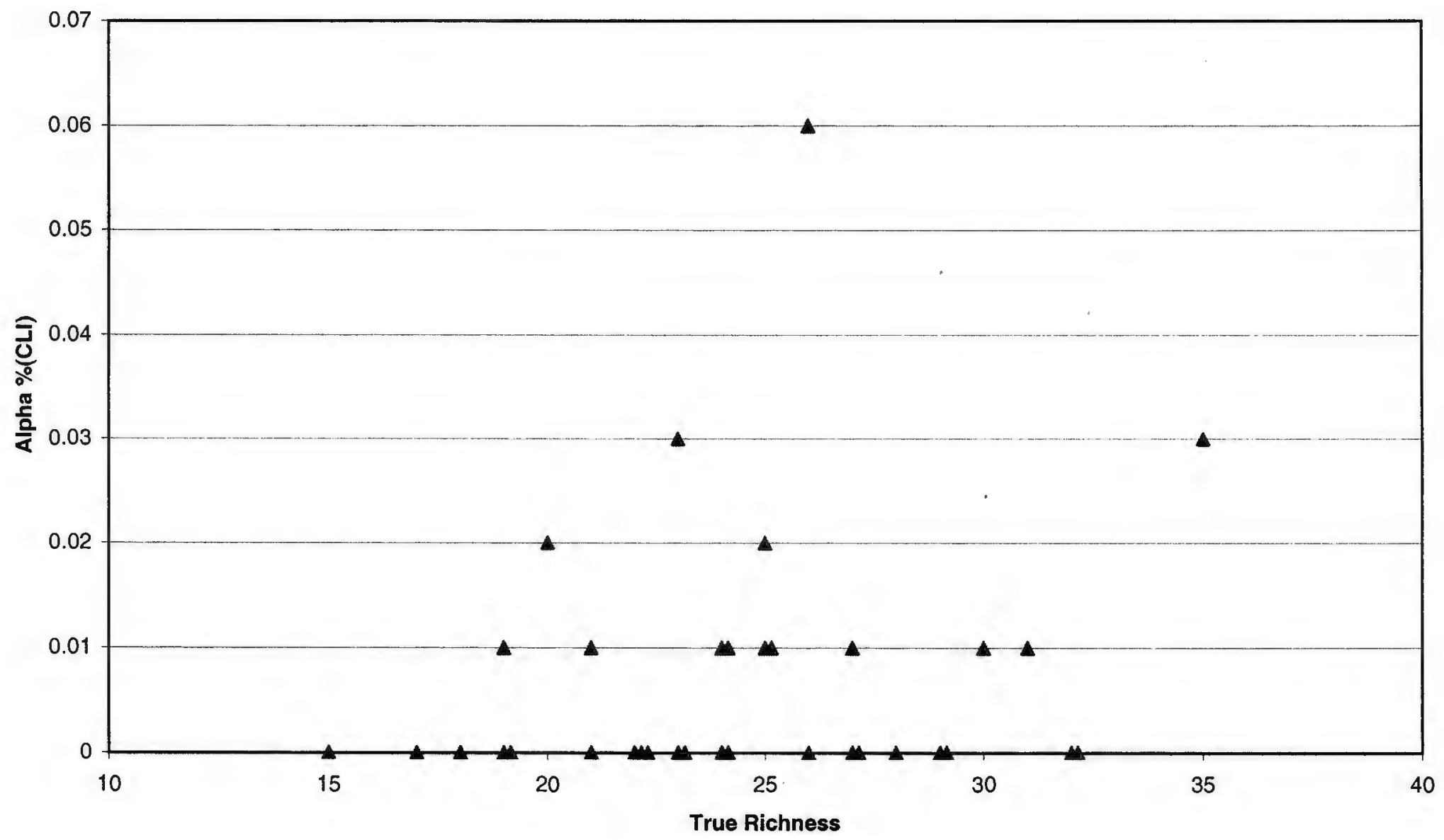




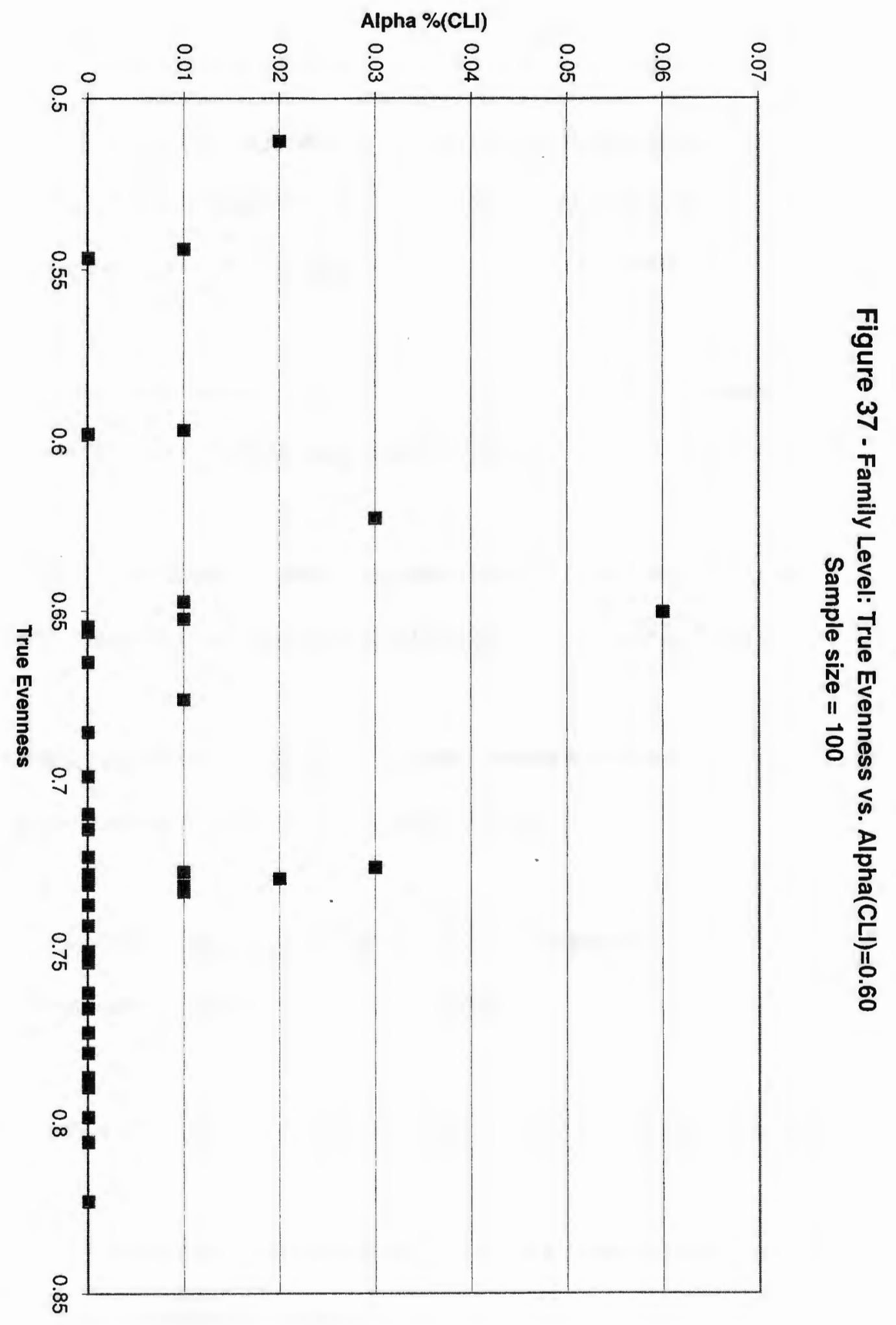




\section{BIBLIOGRAPHY}

Armitage, P.D., D. Moss, J.F. Wright and M.T. Furse (1983). The Performance of a new Biological Water Quality Score System Based on Macroinvertebrates over a Wide Range of Unpolluted Running-Water Sites. Water Research. 17:333-47.

Courtemanche and Davies (1987). A coefficient of community loss to assess detrimental change in Aquatic communities. Water Research. 21(2): 217-222.

Doberstein, Karr, Conquest (2000). The effect of fixed-count subsampling on macroinvertebrates biomonitoring in small streams. Freshwater Biology, 44, 355-371.

Environmental Protection Agency (EPA)(1989). Rapid Bioassessment Protocols for Use in Streams and Rivers, 1-1, 2-2, 6-22, 6-24, 6-25.

Environmental Protection Agency (EPA)(1999). Rapid Bioassessment Protocols for Use in Wadeable Streams and Rivers, 3-1, 3-3, 3-4.

Fager, E.W. (1972). Diversity: a sampling study. American Naturalist 106: 293 - 310.

Krebs, C.J. (1999). Ecological Methodology, Second Edition. Addison-Wellesley Educational Publishers, Inc. 12:412. 
McClave, J.T., Dietrich, F.H., and T. Sincich (1997), Statistics, Seventh Edition, Prentice-Hall, Inc. 8:291.

Olsgard F., P. Somerfield, and M. Carr (1997). Relationships between taxonomic resolution and data transformations in analyses of a macrobenthic community along an established pollution gradient. Marine Ecology Progress Series, Vol. 149: 173-181.

Pielou, E.C. (1966). The measurement of diversity in different types of biological collections. Journal of Theoretical Biology. 13: 131-144.

Rosenberg, D.M. and V.H. Resh (1993). Introduction to Freshwater Biomonitoring and Benthic Macroinvertebrates in Freshwater Biomonitoring and Benthic Macroinvertebrates. David M. Rosenberg and Vincent H. Resh (eds.). Chapman Hill, New York.x, 488pp.

Szal, G. and R. Issac (Dec. 19, 2000), Environmental Engineers, Personal Communication.

Woller, J. (1996). University of Nebraska-Lincoln, The Basics of Monte Carlo Simulations, URL: http://wwitch.unl.edu/zeng/joy/mclab/mcintro.html, November 2001. 